In press at Emotion

\title{
APPRAISAL SHIFTS DURING REAPPRAISAL
}

Andero Uusberg ${ }^{1}$, Jennifer Yih ${ }^{2}$, Jamie L. Taxer ${ }^{2}$, Nicole M. Christ ${ }^{3}$, Teili Toms ${ }^{1}$, Helen Uusberg ${ }^{1}$, James J. Gross ${ }^{2}$

${ }^{1}$ Institute of Psychology, University of Tartu, Estonia

${ }^{2}$ Department of Psychology, Stanford University, USA

3 Department of Psychology, University of Toledo, USA

\begin{abstract}
How to model the processes involved in regulating emotions via reappraisal? In two studies, we tested whether reappraisal impacts emotions through shifts along appraisal dimensions. In an experimental Study 1, 437 students imagined reliving a recent distressing event and rated their appraisals and emotions before and after using reappraisal to feel less negative about the event. Between $19 \%$ and $49 \%$ of changes to different emotions were statistically mediated by shifts along ten appraisal dimensions. Latent profile analyses suggested that the appraisal shifts reflected four distinct reappraisal tactics. These findings were conceptually replicated in an intensive longitudinal Study 2, where 168 participants rated their appraisals and emotions in relation to maximum of 3 emotional events for 7 days, first within an hour of the event and again in the evening when they also reported on emotion regulation use (1142 observations). Between $22 \%$ and $46 \%$ of changes to different emotions accompanying reappraisal use were statistically mediated by shifts along appraisal dimensions. Appraisal shifts were less significant for unregulated and otherwise regulated emotion changes. Relative to Study 1 , the latent profile analyses of Study 2 revealed two similar and four novel reappraisal tactics reflecting a broader range of events and feelings. Across both studies, all appraisal dimensions were involved in at least one tactic and no dimension in all of them, highlighting the suitability of multivariate profiles over univariate dimensions for modelling reappraisal. These findings suggest that appraisal shift profiles can be part of a useful model of cognitive processes underlying reappraisal.
\end{abstract}

Keywords: reappraisal, appraisal shift profiles, emotion regulation, cognitive change ${ }^{1}$

Faced with an unwanted emotion, people often engage in reappraisal by changing how they think about the situation that has elicited the emotion (McRae, 2016). For instance, an insulting comment feels less insulting if one considers that people tend to say things they do not mean during a quarrel. One unresolved question in reappraisal research is how to systematically model the cognitive processes involved in different forms of reappraisal. We have recently proposed that this can be done by representing instances of reappraisal as profiles of shifts along abstract appraisal dimensions that characterize what the situation means for salient motives (Uusberg et al., 2019). In this paper, we report a pair of complementary studies that test two propositions arising from this idea. First, that reappraisal-induced changes to emotions are statistically mediated by shifts along appraisal dimensions. Second, that appraisal shift profiles provide a succinct coordinate system for characterizing instances of reappraisal.

\footnotetext{
${ }^{1}$ Supplemental Information, survey texts, data, and analysis code can be found at https://osf.io/ugfrh/. This study was supported by Estonian Research Council grants PUTJD-79 and PSG525. We are grateful to Craig Smith and Sylvia Kreibig for their advice and insights.
} 


\section{The Role of Appraisal Shifts in Reappraisal}

Reappraisal involves intentional changes to thinking about an emotional situation (Gross, 2015). However, it is less clear which thoughts have the power to change emotions and which ones do not. Likewise, it is unclear how to succinctly model different kinds of thoughts that count as reappraisal. A better understanding of the cognitive mechanisms of reappraisal would not only help resolve such conceptual issues but would also have practical value. It could, for instance, help design interventions that facilitate reappraisal use, which remains low in some situations such as facing high intensity emotions (Sheppes, 2014; Suri et al., 2018) and for some people such as individuals with increased risk for mental health issues (Gross \& John, 2003; Suri et al., 2014).

The reAppraisal framework (Uusberg et al., 2019) seeks to explain the cognitive mechanisms of reappraisal using insights from appraisal theory (Scherer et al., 2001). According to the framework, emotions change under reappraisal when people change how they construe a situation (reappraisal through reconstrual) and when they change the goals and other motives used to evaluate that construal (reappraisal through repurposing; Wang et al., 2021). These changes to construals and motives then go on to alter emotions by producing shifts along appraisal dimensions.

Appraisal dimensions characterize what a situation means for salient motives. Given that motivational meaning is relatively abstract, much of it can be represented using only a few dimensions, such as the following five that many authors consider necessary (Moors et al., 2013). Relevance concerns how much the situation matters for salient motives. Congruence concerns how much the situation helps or hinders pursuing these motives. Accountability concerns whether responsibility for the situation lies with oneself, with someone else, or with no one in particular. Certainty concerns how clear the situations and its implications are. Finally, control concerns the likelihood that one can change or emotionally manage the situation.

The reAppraisal framework suggests that instances of reappraisal manifest as combinations of shifts along such appraisal dimensions. For instance, realizing that people say things they don't mean during a quarrel can make one feel less insulted by lowering how motivationally relevant the situation is appraised to be as well as how accountable the other person is appraised to be for their comment.

To test if reappraisal indeed impacts emotions through appraisal shifts, we assessed two propositions that follow from this idea. According to our first proposition, the effects of successful reappraisal on emotion should be mediated by shifts along appraisal dimensions. Despite being implied by many definitions of reappraisal, the idea that appraisal indeed change during reappraisal has received surprisingly little direct empirical attention (Gross, 2015; Smith \& Lazarus, 1993; Yih, Uusberg, et al., 2019). There is evidence that experimental manipulations of appraisals can impact most components of emotion (Scherer \& Moors, 2019). There is also evidence that when instructed to reappraise, people end up changing most components of emotions (Buhle et al., 2013; McRae, 2016; Webb et al., 2012). What is missing, however, is a linking of these two steps: evidence that when instructed to reappraise, people generate appraisal shifts that are instrumental for changing emotions. The first aim of the current research was to test whether shifts along appraisal dimensions could be a way to generate such evidence.

According to our second proposition, appraisal shifts should provide a succinct coordinate space for characterizing various instances of reappraisal. Reappraisal can be implemented using different cognitive actions or tactics that fall under the definition of reappraisal (McRae et 
al., 2012; Vishkin et al., 2020). However, it is unclear how to identify and organize such tactics. For instance, the lists of tactics found in relevant questionnaires overlap only partially with each-other (Carver et al., 1989; Garnefski \& Kraaij, 2007; Haynes et al., 2009) as well as with the ways participants have been found to reappraisal standardized affective images in the laboratory (McRae et al., 2012).

Appraisal shifts may help solve this problem. Appraisal dimensions represent the motivational essence of a situation that abstracts away much of the details. For instance, different situations that elicit fear tend to share a common appraisal profile of high relevance, low congruence, and low control. We propose that appraisal shift profiles may similarly represent the essence of a reappraisal while abstracting away the idiographic variation in specific construal and motive changes people initiate during reappraisal. The second aim of this research was to test this idea by asking if appraisal shift profiles form clusters that resemble meaningful reappraisal tactics.

\section{The Present Research}

We investigated the role of appraisal shifts in reappraisal in two complementary studies that strike different balances between experimental control, measurement granularity, and ecological validity. In a first experimental study, 437 students imagined reliving a recent negative emotional event and then characterized it in terms of emotions and appraisals before and after being instructed to reappraise the event. In a second intensive longitudinal study, 168 participants characterized the strongest emotional event within the last hour in terms of emotions and appraisals up to 3 times a day for 7 days. Each evening, they reported their current feelings and appraisals about each of the daily events along with any intervening attempts to use reappraisal and other emotion regulation strategies.
In both datasets, we first tested the hypothesis that reappraisal-induced emotion changes were statistically mediated by appraisal shifts. Using structural equation models (SEM), we asked whether the extents to which emotions changed after instructed (Study 1) and spontaneous (Study 2) reappraisal were statistically mediated by the extent to which appraisals shifted. These analyses fall short of testing whether appraisal shifts are mechanistic mediators of the effects of upstream thought changes on emotions. Testing this causal claim is virtually impossible with self-reports as it would require assessing appraisals before they lead to emotion changes and emotion changes only after that (Fiedler et al., 2011). Therefore, our aim was to learn whether appraisal sifts are statistical mediators of a correlation between reappraisal and emotion change. Evidence of statistical mediation would be a useful first step towards building a stronger case for the proposed mechanistic role of appraisal shifts in reappraisal.

In both datasets, we also examined whether appraisal shift profiles could reveal distinct reappraisal tactics. We used latent profile analysis (LPA) to ask whether appraisal shift profiles form identifiable clusters and whether these clusters represent distinct and realistic forms of reappraisal. Given that the present studies involved different and limited ranges of emotions and participant populations, we did not expect to find the same reappraisal tactics across studies nor to recover a generalizable taxonomy of tactics. Our goal was to test the potential of appraisal shift profiles to reveal different reappraisal tactics.

\section{STUDY 1: REAPPRAISING RELIVED EVENTS}

In this study, we tested if appraisal shifts underlie different forms of reappraisal using granular self-reports about reactivated emotions collected in an experimental design. In 
an online emotion reactivation and reappraisal task, participants imagined reliving a recent negative emotional event and described their appraisals and emotional reactions before as well as after being instructed to use reappraisal to feel less negative about the event. We focused on down-regulation of negative emotions to avoid conflating variance in reappraisal with variance in emotion regulation goals. We focused on the past two months to capture sufficiently distressing events without introducing overly strong memory biases due to long temporal distance. We used a granular 27item feelings questionnaire and developed and pilot-tested single items to assess 10 dimensions relating to the 5 core appraisals found in most appraisal models.

\section{Method}

\section{Transparency and openness}

We report all details on how sample sizes were determined, all data exclusions, all manipulations, and all measures used in the study. The Supplemental Information alongside with study materials, preprocessed data, and analysis code can be found at https://osf.io/ugfrh/. The study design and its analyses were not preregistered. Analyses were conducted in R ( $R$ Core Team, 2014) with the RStudio platform (RStudio Team, 2015) and the tidyverse set of packages (Wickham et al., 2019). We used the psych package (Revelle, 2014) for exploratory factor analyses, the lavaan package (Rosseel, 2012) for SEM, and the tidyLPA package (Rosenberg et al., 2018) for LPA. We used the Benjamini \& Hochberg method for False Discovery Rate (FDR) corrections. We interpret effect sizes following updated conventions suggested by Funder and Ozer (2019).

\section{Participants}

Students from different San Francisco Bay Area colleges participated online in exchange for course credit in 2018. Data were collected during one semester from all eligible volunteers.
No specific sample size target was determined, and no analyses were conducted before data collection was terminated by the end of the semester. The final sample included 437 participants out of 512 students who completed the study based on correct responses to four attention check questions embedded throughout the study. Participants were excluded for failing any of four attention check questions that had the form of "If you are paying attention, select "A Lot"' with the response option varying between questions. Of the final sample, $71.4 \%$ were female, $26.6 \%$ male, and $2 \%$ of other gender. In terms of race and ethnicity, $34.8 \%$ of participants identified as White, $25.4 \%$ as Asian, $16 \%$ as Hispanic or Latino/Latina, $4.8 \%$ as Black or African American and the remaining $19 \%$ as multiethnic or some other ethnicity. Although the age of participants ranged between 18 and 65 years, most of them were young adults ( $M=$ $22.48, S D=7.33$, 8oth percentile $=23$ ). The study was approved by the Institutional Review Board of Stanford University.

\section{Procedure}

The study was presented online via the Qualtrics survey platform. After confirming informed consent, participants completed the following 8 steps. (1) Recall a distressing event from the past 1-2 months, imagine that it is happening now, and write a few sentences about it. (2) Rate appraisals of the distressing event. (3) Rate emotional reactions to the distressing event. (4) Implement reappraisal. The instructions asked participants to "try to re-evaluate the situation in order to change the emotions it elicits." It provided a pair of examples illustrating reconstrual and repurposing forms of reappraisal and then encouraged participants to pause and "think about how you could have viewed the situation from a different angle, or how you could have re-thought what it really meant." Participants were also asked to write a few sentences about how they thought differently about the event. (5) Rate appraisals 
of the reappraised event. (6) Rate emotional reactions to the reappraised event. (7) Respond to a series of individual difference measures not analyzed in this paper (see Supplemental Information). (8) Read a debriefing text.

\section{Measures}

Emotions. Emotional feelings were measured using 27 items, each comprising up to three adjectives designed to capture a single discrete emotion (Yih, Kirby, et al., 2019). The items were phrased to complete the sentence "In this situation, I feel ...". Participants indicated their agreement with each item on a 9-point scale combining numbers and labels: Strongly Disagree (1) - (2) - A Little (3) - (4) - Moderately (5) - (6) - A Lot (7) - (8) - Strongly Agree (9).

Appraisals. Ten items were designed and pilottested to capture dimensions representing five core appraisals. In a pilot study, described in more detail in the Supplemental Information, 60 students assessed their appraisals and emotions in relation to 6 vignettes that elicited similar emotional responses. Considering the vignettes as repeated measures, we found that intra-class correlations for each appraisal item (two-way model, agreement type, and average units; see Supplemental Table 1 ) ranged from .28 to .87 (M $=.61, \mathrm{SD}=.19$ ) with 7 items above 0.6 indicating moderately reliability (Koo \& Li, 2016). We also confirmed that the appraisal items were related to emotion ratings in a largely expected selective pattern.

Each appraisal item was phrased to complete the sentence "In this situation, I have a sense that ..." corresponding to the task to imagine reliving the recalled distressing event. Participants indicated their agreement with each item on a 9-point scale combining numbers and labels: Strongly Disagree (1) - (2) - (3) - (4) - Neither (5) - (6) - (7) - (8) - Strongly Agree (9).

The items targeted the following 10 dimensions distributed between 5 appraisals. Relevance for goals and motives (... this situation matters to me). Congruence (... this situation is potentially desirable for me) and incongruence (... this situation is potentially harmful for me) with goals and motives. Accountability by the self (... I am responsible for this situation) by others (... someone else is responsible for this situation) and no one (... no one in particular is responsible for this situation). Outlook certainty (... I don't know how this situation is going to turn out). Coping potential on the situational (... I can change this situation for the better) and emotional (... I can accept whatever happens in this situation) levels alongside outlook valence (... one way or another, this situation is going to turn out well). The order of appraisal and emotion items was randomized on each presentation. The appraisal measurement also included six items not analyzed in this paper for reasons explained in the Supplemental Information.

\section{Results and Discussion}

Preliminary analyses

Most events reported by participants were relatively distant in time: $1.6 \%$ of events were from the same day, $14.4 \%$ from the last 7 days, $14.9 \%$ from last 14 days, 30.9\% from the last month, $29.5 \%$ from the last two months, and $8.7 \%$ from more than 2 months ago. Even though participants were not instructed to recall events beyond 2 months, we retained these reports in the data to honor the contributions of all participants.

To test key assumptions of the emotion reactivation and reappraisal task, we analyzed the mean emotion and appraisal ratings from before and after reappraisal (see Figure 1 for the means, Cohen's d effect sizes, and single sample t-test p-levels of each change; see Supplemental Table 2 for the numeric values of the depicted means and their standard deviations). Indicating successful emotion elicitation, the events participants recalled were rated relatively high on negative emotions and low on positive emotions. Indicating successful 
reappraisal manipulation, all ratings of negative emotions were significantly lower and ratings of positive emotions significantly higher after compared to before reappraisal. Improvements in negative emotions were generally larger than improvements in positive emotion.

Successful emotion elicitation was also indicated by appraisal ratings. Mean ratings were most extreme on the appraisals that should be common to most negative emotions: high relevance, high incongruence, and low congruence. Indicating successful reappraisal manipulation, mean appraisal ratings collected

A

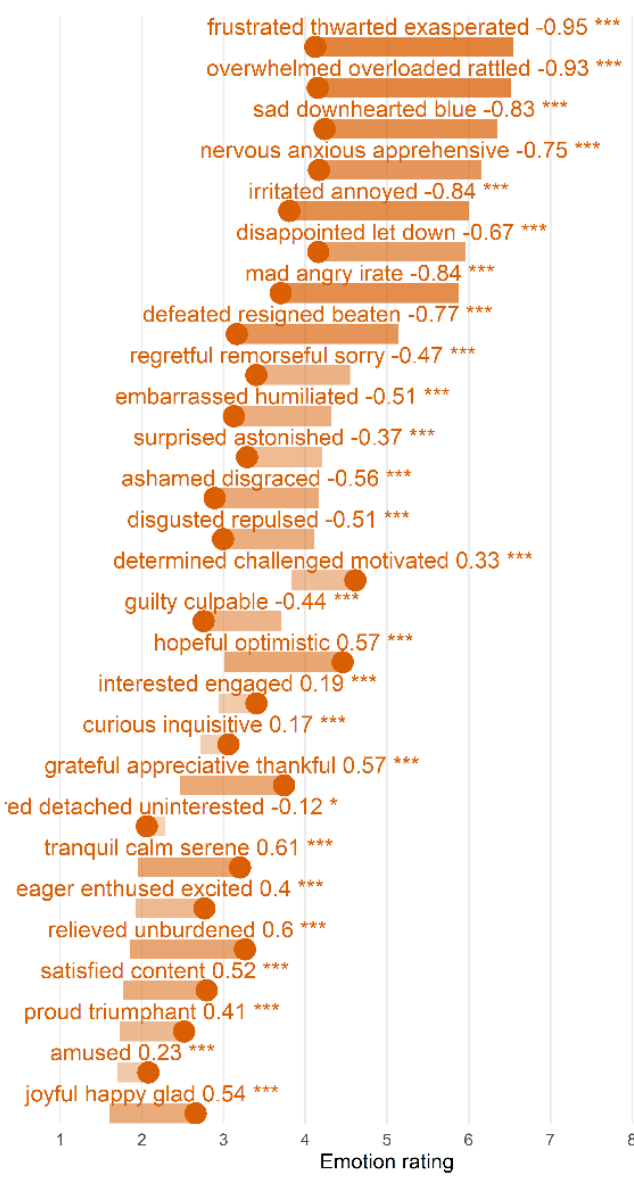

before and after regulation differed significantly for all dimensions other than selfaccountability. On average, after following the reappraisal instruction, participants reported lower relevance, incongruence, outlook certainty, and other accountability appraisals. They reported higher situational coping, emotional coping, outlook valence, impersonal accountability, and congruence appraisals. Appraisal shifts were generally of smaller magnitude than emotion changes. Taken together, the mean emotion and appraisal ratings confirm that the emotion reactivation and reappraisal task functioned as intended.

B

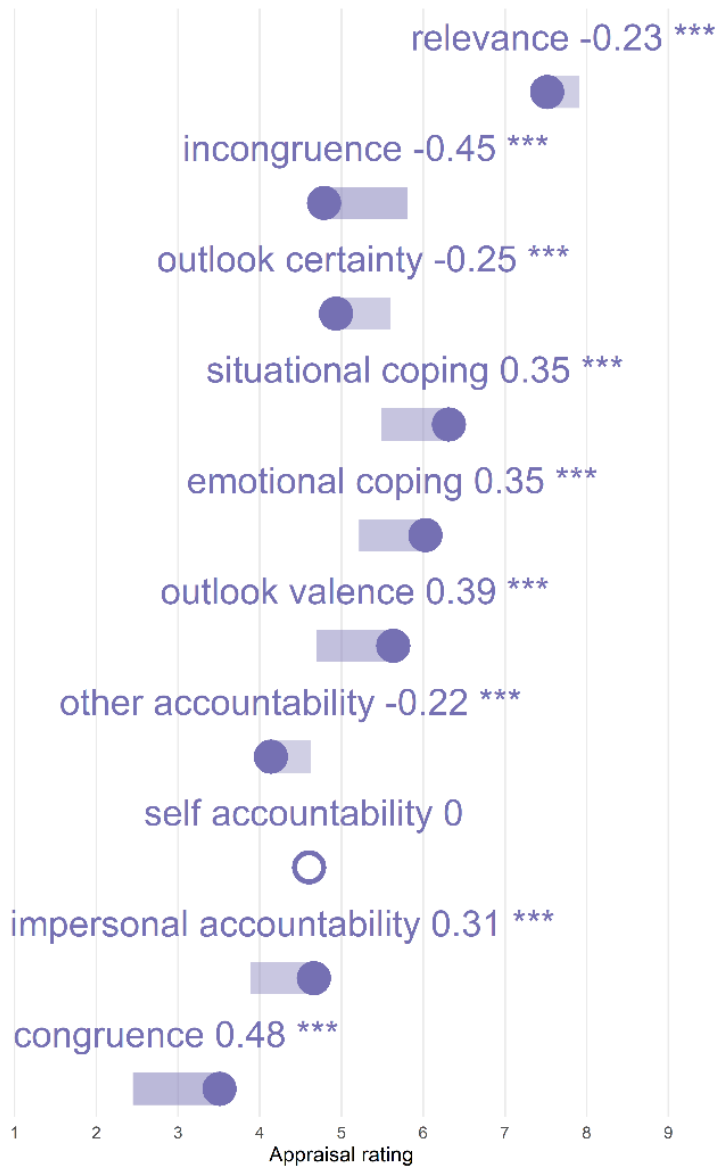

Figure 1. Mean emotion changes (panel A) and appraisal shifts (panel B) from before to after using reappraisal.

Notes. The non-pointed beginning of each line depicts the pre-regulation value and the pointed end the post-regulation value on original response scales. Lines are ordered by pre-regulation values. The numbers depict Cohen's $d$ effect sizes with uncorrected $\mathrm{p}$-values from $\mathrm{t}$-tests. Filled points depict significant effects and the stars denote significance levels $* * * \mathrm{p}<.001$, $* * p<.01, * p<.05$. 
As many emotion items were significantly intercorrelated, we used an exploratory factor analysis to represent their variance in a more parsimonious manner while retaining sensitivity to discrete emotions. Factors were identified from the pre-regulation ratings to avoid contamination by reappraisal. Five factors suggested by parallel analysis were extracted using the principal axis method and oblimin rotation yielding a model with a good fit (TLI = 0.89 , RMSEA = 0.056). The factors were labelled based on item loadings as satisfied, irritated, determined, ashamed, and overwhelmed (see Supplemental Table 3 for factor loadings and proportions explained). For subsequent analyses, we computed factor scores with the regression method for both pre- and postregulation ratings (factor score $0=$ sample mean across pre- and post-regulation).
Were reappraisal effects on emotion statistically mediated by appraisal shifts?

Turning to our hypothesis, we tested whether the emotion changes we observed were statistically mediated by the appraisal shifts we observed. Specifically, we conducted a twocondition within-subject mediation analysis for each emotion factor (Montoya \& Hayes, 2017). In each model (see Figure 2, panel A), 10 shifts on appraisal dimensions from pre- to postregulation were entered as parallel mediators of a change in the emotion factor score controlling for sample-centered mean appraisals. Detailed results of the mediation analyses can be found in Table 1. Panel B on Figure 2 summarizes the key findings by depicting the proportions of change in each emotion that was statistically mediated by appraisal shifts.
A

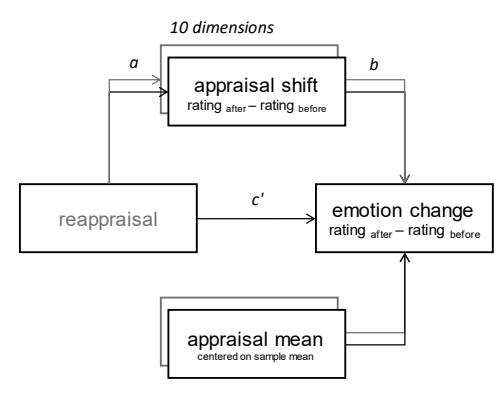

B

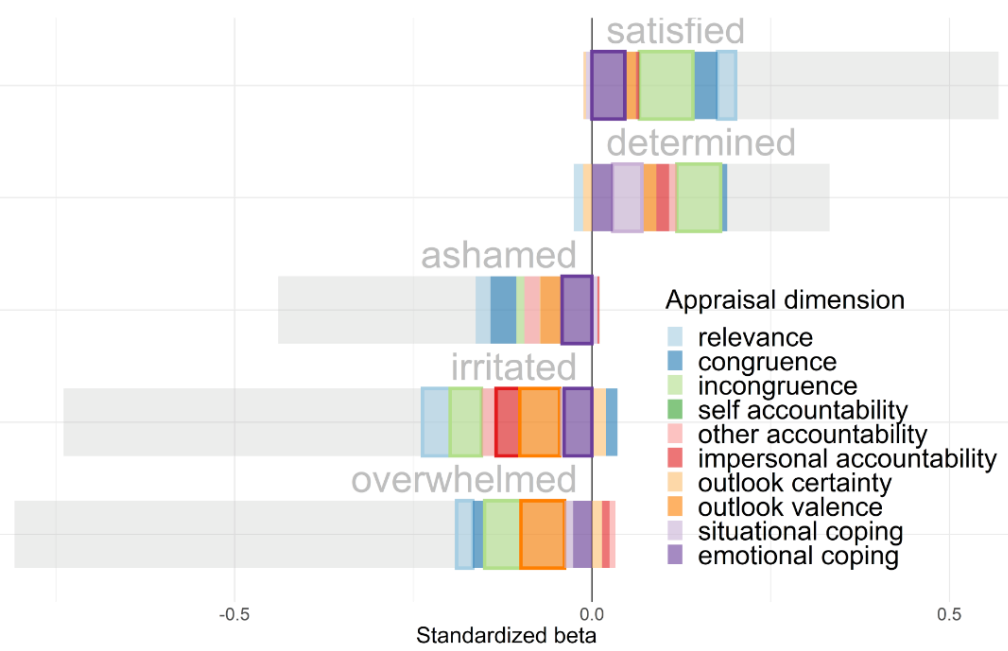

Figure 2. Structure and main results of the mediation analyses. Panel A. The two-condition within-subject mediation model. Panel B. Proportions of reappraisal-related emotion changes statistically mediated by appraisal shifts.

Notes. Panel A. In the model, emotion change defined as the difference between post and pre emotion factor scores is predicted by 10 appraisal shifts defined as differences between post and pre appraisal ratings; an intercept representing the direct effect of reappraisal; and 10 appraisal means defined as a sample centered average of pre and post ratings to make the intercept interpretable as a direct effect. Panel B. Gray bars represent total emotion changes from pre to post regulation. Colored ribbons represent proportions of these changes that were statistically mediated by appraisal shifts. Ribbons with thick outlines denote significant effects at uncorrected $\mathrm{p}<.05$. 
Table 1. Standardized path coefficients from five within-subject mediation analyses.

\begin{tabular}{|c|c|c|c|c|c|c|c|c|c|c|}
\hline & \multicolumn{2}{|c|}{ Overwhelmed } & \multicolumn{2}{|c|}{ Irritated } & \multicolumn{2}{|c|}{ Ashamed } & \multicolumn{2}{|c|}{ Determined } & \multicolumn{2}{|c|}{ Satisfied } \\
\hline & $\beta$ & $p$ & $\beta$ & $p$ & $\beta$ & $p$ & $\beta$ & $p$ & $\beta$ & $p$ \\
\hline \multicolumn{11}{|l|}{ Appraisal shifts (a paths) } \\
\hline Relevance & -0.234 & $<0.001$ & -0.234 & $<0.001$ & -0.234 & $<0.001$ & -0.234 & $<0.001$ & -0.234 & $<0.001$ \\
\hline Congruence & 0.478 & $<0.001$ & 0.478 & $<0.001$ & 0.478 & $<0.001$ & 0.478 & $<0.001$ & 0.478 & $<0.001$ \\
\hline Incongruence & -0.452 & $<0.001$ & -0.452 & $<0.001$ & -0.452 & $<0.001$ & -0.452 & $<0.001$ & -0.452 & $<0.001$ \\
\hline Self-accountability & 0.002 & 0.964 & 0.002 & 0.964 & 0.002 & 0.964 & 0.002 & 0.964 & 0.002 & 0.964 \\
\hline Other-accountability & -0.220 & $<0.001$ & -0.220 & $<0.001$ & -0.220 & $<0.001$ & -0.220 & $<0.001$ & -0.220 & $<0.001$ \\
\hline Impersonal accountability & 0.308 & $<0.001$ & 0.308 & $<0.001$ & 0.308 & $<0.001$ & 0.308 & $<0.001$ & 0.308 & $<0.001$ \\
\hline Outlook valence & 0.394 & $<0.001$ & 0.394 & $<0.001$ & 0.394 & $<0.001$ & 0.394 & $<0.001$ & 0.394 & $<0.001$ \\
\hline Outlook certainty & -0.253 & $<0.001$ & -0.253 & $<0.001$ & -0.253 & $<0.001$ & -0.253 & $<0.001$ & -0.253 & $<0.001$ \\
\hline Situational coping & 0.350 & $<0.001$ & 0.350 & $<0.001$ & 0.350 & $<0.001$ & 0.350 & $<0.001$ & 0.350 & $<0.001$ \\
\hline Emotional coping & 0.355 & $<0.001$ & 0.355 & $<0.001$ & 0.355 & $<0.001$ & 0.355 & $<0.001$ & 0.355 & $<0.001$ \\
\hline \multicolumn{11}{|l|}{ Appraisal effects on emotion (b paths) } \\
\hline Relevance & 0.097 & 0.030 & 0.164 & $<0.001$ & 0.089 & 0.049 & 0.055 & 0.210 & -0.109 & 0.012 \\
\hline Congruence & -0.034 & 0.443 & 0.033 & 0.442 & -0.076 & 0.095 & 0.019 & 0.663 & 0.072 & 0.096 \\
\hline Incongruence & 0.113 & 0.012 & 0.097 & 0.024 & 0.025 & 0.588 & -0.136 & 0.002 & -0.166 & $<0.001$ \\
\hline Self-accountability & -0.002 & 0.965 & -0.135 & 0.002 & 0.056 & 0.217 & 0.065 & 0.139 & 0.082 & 0.058 \\
\hline Other-accountability & -0.036 & 0.424 & 0.092 & 0.032 & 0.097 & 0.033 & -0.050 & 0.257 & 0.002 & 0.967 \\
\hline Impersonal accountability & 0.034 & 0.446 & -0.108 & 0.012 & 0.009 & 0.849 & 0.057 & 0.196 & 0.016 & 0.716 \\
\hline Outlook valence & -0.154 & 0.001 & -0.141 & 0.001 & -0.078 & 0.087 & 0.052 & 0.239 & 0.039 & 0.370 \\
\hline Outlook certainty & -0.056 & 0.211 & -0.079 & 0.067 & 0.004 & 0.924 & 0.049 & 0.262 & 0.012 & 0.790 \\
\hline Situational coping & -0.035 & 0.440 & -0.018 & 0.676 & 0.022 & 0.625 & 0.116 & 0.008 & -0.024 & 0.574 \\
\hline Emotional coping & -0.074 & 0.098 & -0.109 & 0.011 & -0.117 & 0.010 & 0.082 & 0.061 & 0.130 & 0.003 \\
\hline \multicolumn{11}{|l|}{ Mediated emotion change (a*b) } \\
\hline Relevance & -0.023 & 0.048 & -0.038 & $0.003^{*}$ & -0.021 & 0.068 & -0.013 & 0.224 & 0.025 & 0.026 \\
\hline Congruence & -0.016 & 0.444 & 0.016 & 0.443 & -0.036 & 0.100 & 0.009 & 0.663 & 0.035 & 0.101 \\
\hline Incongruence & -0.051 & 0.015 & -0.044 & 0.029 & -0.011 & 0.589 & 0.061 & $0.003^{*}$ & 0.075 & $<0.001^{*}$ \\
\hline Self-accountability & -0.000 & 0.975 & -0.000 & 0.964 & 0.000 & 0.964 & 0.000 & 0.964 & 0.000 & 0.964 \\
\hline Other-accountability & 0.008 & 0.431 & -0.020 & 0.052 & -0.021 & 0.053 & 0.011 & 0.271 & -0.000 & 0.967 \\
\hline Impersonal accountability & 0.011 & 0.449 & -0.033 & 0.020 & 0.003 & 0.849 & 0.017 & 0.205 & 0.005 & 0.717 \\
\hline Outlook valence & -0.061 & $0.001^{*}$ & -0.056 & $0.002^{*}$ & -0.031 & 0.094 & 0.020 & 0.243 & 0.015 & 0.373 \\
\hline Outlook certainty & 0.014 & 0.224 & 0.020 & 0.083 & -0.001 & 0.924 & -0.012 & 0.272 & -0.003 & 0.790 \\
\hline Situational coping & -0.012 & 0.442 & -0.006 & 0.676 & 0.008 & 0.625 & 0.041 & 0.013 & -0.009 & 0.575 \\
\hline Emotional coping & -0.026 & 0.107 & -0.039 & $0.016^{*}$ & -0.042 & 0.015 & 0.029 & 0.070 & 0.046 & $0.006^{*}$ \\
\hline \multicolumn{11}{|l|}{ Total effects } \\
\hline Total mediated emotion change & -0.157 & $0.001^{*}$ & -0.201 & $<0.001^{*}$ & -0.152 & $0.002^{*}$ & 0.164 & $0.001^{*}$ & 0.189 & $<0.001^{*}$ \\
\hline Proportion mediated & 0.194 & & 0.272 & & 0.347 & & 0.493 & & 0.333 & \\
\hline Unmediated emotion change (c') & -0.651 & $<0.001$ & -0.538 & $<0.001$ & -0.287 & $<0.001$ & 0.168 & 0.008 & 0.380 & $<0.001$ \\
\hline Total emotion change & -0.808 & $<0.001$ & -0.739 & $<0.001$ & -0.439 & $<0.001$ & 0.332 & $<0.001$ & 0.569 & $<0.001$ \\
\hline
\end{tabular}

Notes. $\beta$ - standardized regression coefficient, $p$ - uncorrected significance value. Bold denotes significant effects at uncorrected $\mathrm{p}<0.05$ * denotes $\mathrm{p}$-values that remained significant after applying the FDR-correction to the mediation effects on gray background that were relevant for our hypothesis. 
Among negative emotions, appraisal shifts statistically mediated $19 \%(b=-.16, p=.001)$ of the reappraisal-related reduction in feeling overwhelmed, $b=-.81, p<.001$. This emotion change was related to a significant increase in the outlook valence appraisal (accounting for $7.5 \%$ of the emotion change, $p=.001$ ) and significant decreases in incongruence $(6.3 \%, p=$ $.015)$ as well as relevance $(2.8 \%, p=.048)$ appraisals. Appraisal shifts also statistically mediated $27 \%(B=-.20, p<.001)$ of the reappraisal-related reduction in feeling irritated, $b=-.74, p<.001$. This emotion change was related to significant increases in outlook valence $(7.5 \%, p=.002)$, emotional coping $(5.3 \%, p=.016)$, and impersonal accountability $(4.5 \%, p=.020)$ appraisals as well as significant decreases in incongruence $(5.9 \%, p=.029)$ and relevance $(5.2 \%, p=.003)$ appraisals. Finally among negative emotions, appraisal shifts statistically mediated $35 \%(b=-.15, p=.002)$ of the reappraisal-related reduction in feeling ashamed, $b=-.44, p<.001$. This emotion change was related to a significant increase in the emotional coping appraisal $(9.5 \%, p=.015)$.

Appraisal shifts also statistically mediated reappraisal effects on positive emotions. Appraisal shifts mediated $49 \%(b=.16, p=.001)$ of the reappraisal-related increase in feeling determined, $b=.33, p<.001$. This emotion change was related to a significant decrease in incongruence appraisal $(19 \%, p=.006)$ and a significant increase in situational coping appraisal (12\%, $p=.017)$. Appraisal shifts also mediated $33 \%(b=.19, p<.001)$ of the reappraisal-related increase in feeling satisfied, $b=.57, p<.001$. This emotion change was related to significant decreases in incongruence $(13.1 \%, p=.001)$ and relevance $(4.5 \%, p=.027)$ appraisals as well as a significant increase in emotional coping appraisal $(8.1 \%, p=.006)$.

Taken together, the SEM results support our hypothesis that reappraisal-related changes to emotion are statistically mediated by shifts along appraisal dimensions. Between different emotions, all appraisal shifts combined had small-to-medium relationships with emotion changes and explained 19 to $49 \%$ of emotion change variance. These effects were relatively robust. When we applied the FDR correction to the 55 mediation effects in Table 1, all total mediation effects remained significant along with seven of the 14 effects of individual appraisal dimensions.

Can appraisal shift profiles reveal reappraisal tactics?

If appraisal shift profiles can indeed succinctly represent different instances of reappraisal in a common coordinate space, then the profiles observed in this study should form meaningful clusters rather than be uniformly distributed. We tested this idea by comparing LPA models that attribute the observed appraisal shift profiles to a different number of latent classes. As an input to the LPA, we used residualized difference scores (i.e., residuals from regressions where a pre-regulation score predicted a post-regulation score) to reduce the measurement error associated with simply subtracting the pre- and post-regulation scores (Castro-Schilo \& Grimm, 2018). LPA models used the maximum likelihood estimator and indicator variances and covariances were estimated separately for each class. In line with our expectation, we found that appraisal shift profiles were best explained by an LPA model with more than one class. Specifically, the Adjusted Lo-Mendell-Rubin (LMR) Likelihood Ratio test (Nylund et al., 2007) suggested that a model with four classes fit the observed appraisal shift profile variance significantly better than models with fewer classes $(p<.001)$ whereas a model with five classes did not provide further significant improvements $(p=$ .18, see Supplemental Table 4 for other model fit statistics). 


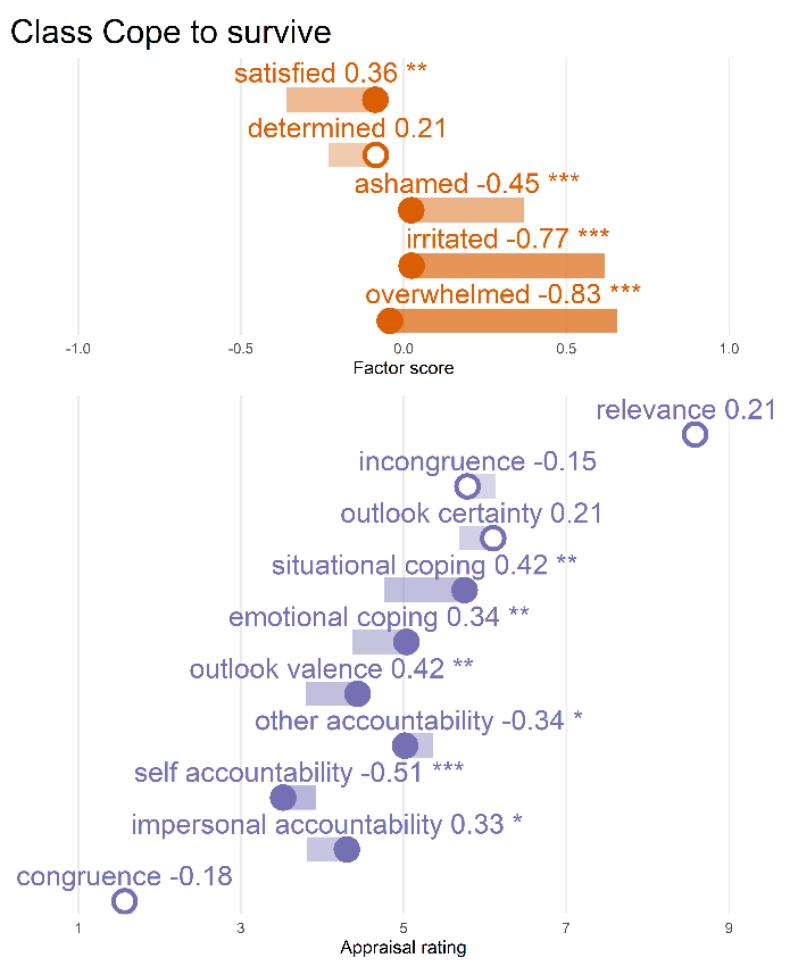

\section{Class Let it go}

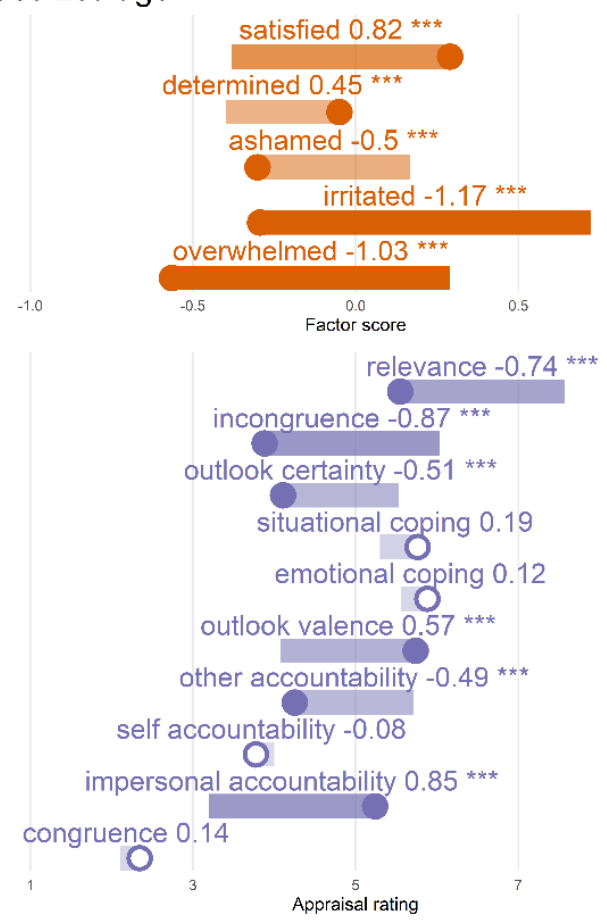

\section{Class Cope to thrive}

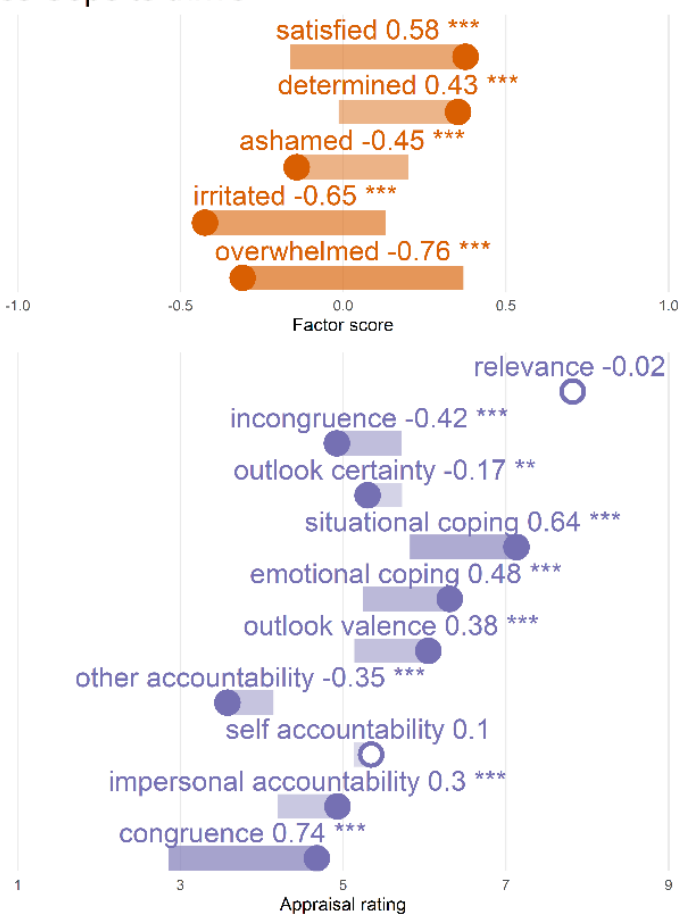

\section{Class You never know}

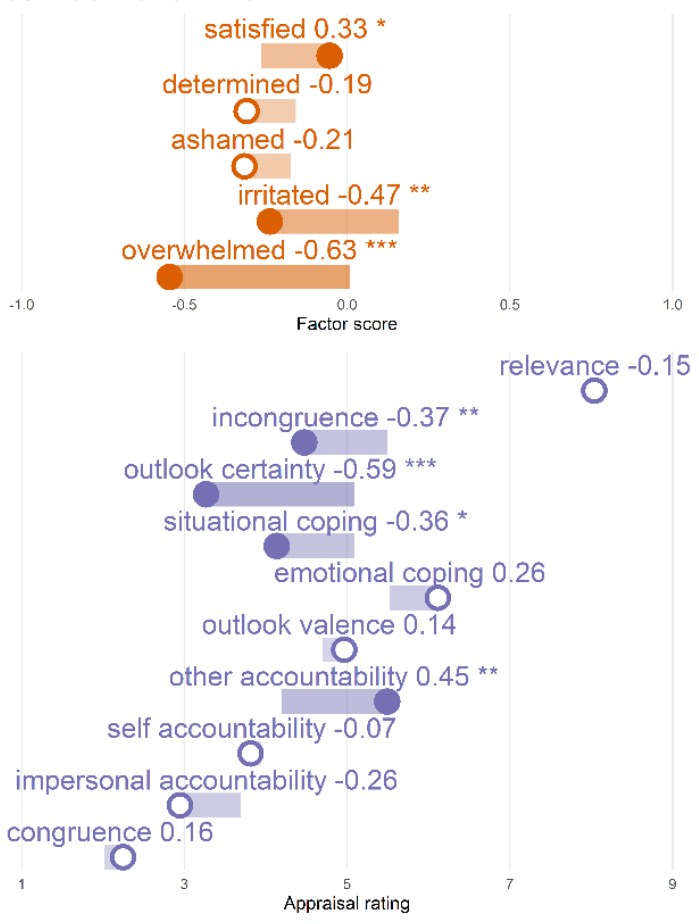

Figure 3. Mean emotion changes and appraisal shifts within four reappraisal classes.

Notes. The non-pointed beginning of each line depicts the pre-regulation value and the pointed end the post-regulation value. The lines are ordered by pre-regulation values. The numbers depict Cohen's $d$ effect sizes with uncorrected $p$-values. Filled points depict significant effects and the stars denote significance levels $* * * p<.001, * * p<.01, * p<.05$. Emotion changes are on factor scores scales ( $\mathrm{o}=$ sample mean across pre- and post-regulation). Appraisal shifts are on the original response scale. 
The idea that appraisal shift profiles capture distinct forms of reappraisal was also supported by the face validity of the appraisal shifts and emotion changes characteristic of each LPA class that can be seen in Figure 3. We labelled Class $1(n=60)$ "Cope to survive". Coping was implied by shifts in the future oriented appraisals of situational coping, emotional coping, and outlook valence. The tactic also involved a shift away from blaming oneself and to a lesser extent from blaming others toward impersonal accountability suggesting a refocusing from past to the future. Surviving rather than thriving was implied by an absence of shifts in congruence and incongruence. Also, this tactic involved relatively intense negative emotions changing to no further than the sample mean and only a modest increase in satisfaction.

We labelled Class 2 ( $n=240$ ) "Cope to thrive". Coping was again implied by significant increases in the same future-oriented appraisals as well as shifting from past-oriented blaming of others toward impersonal accountability. Thriving was implied by increased congruence and reduced incongruence appraisals as well as by notable increases in positive emotion as well as reductions in negative emotion below sample mean, albeit from a less intense starting point.

We labelled Class $3(n=84)$ "Let it go". Disengagement was implied by reduced relevance, seen only in this tactic, coupled with reduced incongruence. This tactic also involved a more hopeful yet less certain outlook for the future as well as shifting blame away from others. This tactic was accompanied by marked reductions in negative emotions, particularly feeling overwhelmed and irritated, as well as increases in positive emotions, particularly satisfaction.

We labelled Class $4(n=53)$ "You never know". A potential for a positive outcome was implied by a reduced outlook certainty and reduced incongruence. The tactic also involved a unique combination of increased other accountability and reduced situational coping suggesting reduced agency. The tactic was accompanied by reductions in feeling overwhelmed and irritated from relatively mild starting points without major increases in positive emotion.

\section{Interim summary}

Findings from Study 1 largely supported both of our propositions. First, we found that shifts along appraisal dimensions statistically mediated significant portions of the impact of using reappraisal on emotion ratings. This is consistent with, although it does not prove, the idea that appraisal shifts are instrumental to converting the more specific thought changes that constitute reappraisal into emotion changes, as suggested by the reAppraisal framework.

Second, we found that appraisal shift profiles formed distinct classes that may represent meaningful reappraisal tactics. We also found that successful reappraisals can involve appraisal shifts in different directions. Some appraisals were shifted in the same direction in all tactics - relevance and incongruence were reduced and outlook valence and emotional coping were increased. Situational coping and all accountability dimensions by contrast shifted in different directions across different tactics. This pattern further underscores the value of considering a profile of appraisals as a useful map of reappraisal.

The inferences of Study 1 are limited in several ways, two of which were addressed in Study 2. First, the findings are open to cognitive distortions. Inevitably, appraisals of and emotional reactions to recalled and mentally simulated events such as the ones studied here differ from concurrent measurements. The findings could have also been biased by demand characteristics as the reappraisal instruction made it clear what was being studied and preand post-regulation data were collected in close temporal proximity. Second, emotions can 
change through shifting appraisals for reasons other than reappraisal (Lazarus, 1968; Yih, Uusberg, et al., 2019). It is thus possible that the mediation effects we observed reflect appraisals shifting for reasons other than the reappraisal manipulation we employed. Study 2 enables us to remedy both of these limitations.

\section{STUDY 2: REAPPRAISING DAILY EVENTS}

In Study 2, we sought to conceptually replicate findings of Study 1 while addressing some of its limitations using an intensive longitudinal design. For 7 consecutive days, participants were asked at around 11:00, 13:00 and 15:00 o'clock to characterize the emotions, appraisals, and context of the most intense emotional event of the past hour. At 19:00 o'clock they were asked to think back to the events they reported, characterize them again, and also report interim attempts at emotion regulation. This design allowed us to address a pair of limitations of Study 1. Collecting pre-regulation assessments during the day no later than one hour after an emotional event reduced the potential for memory biases to affect the emotion reports. Meanwhile, collecting postregulation data at least two hours and usually several hours after the pre-regulation data alleviated the impact of demand characteristics as participants were unlikely to remember their pre-regulation ratings. Second, in this study we could compare appraisal-mediated emotion changes that accompany reappraisal to appraisal-mediated emotion changes that accompany use of other emotion regulation strategies or no strategy at all.

\section{Method}

\section{Transparency and openness}

We follow the same principles and procedures as in Study 1.

\section{Participants}

Participants were recruited using social media and University of Tartu mailing lists form Estonian speaking adults in possession of a smart phone. The data were collected for two weeks in 2019, no target sample size was determined. The sample analyzed here includes 168 people who provided at least one pair of day and evening reports out of 189 initial participants. Based on 121 participants who also provided demographic data, the sample was between 18 and 52 years of age $(M=28.8 ; S D=$ 7.8), $84.3 \%$ female and $93.4 \%$ higher educated (6.6\% had secondary education). At the end of the study, participants received feedback about their Big Five personality traits and correlations between the appraisal shifts and emotion changes they reported. They also participated in a raffle of three $50 €$ bookstore gift cards. The study was approved by the Institution Ethics Review Board of University of Tartu.

\section{Procedure}

After confirming informed consent and providing their contact information, participants viewed an online video tutorial explaining study logistics and the day and evening surveys. They were then directed to an online individual difference survey they could fill in at any time (data not analyzed here, see Supplemental Information for measures).

For 7 days starting from the day after completing the video tutorial, participants received 4 text messages a day containing a link to a Qualtrics survey they could fill in using their smartphone browser. The messages were composed using a Google Sheets script and sent through the $46 \mathrm{elks}$.com service. The messages sent at about 11:00, 13:00 and 15:00 o'clock led to a daily survey whereas the message sent at about 19:00 o'clock led to an evening survey. We elected to send messages at the same time to everyone to limit differences in the temporal distance between the day and evening surveys. Participants had 2 hours to respond to each daily 
survey and until the next morning to respond to the evening survey. If they clicked the link outside of these times, they were directed to the next survey or shown an end of survey message.

\section{Measures}

To maintain high response rates, the day and evening surveys were designed to require minimal time and effort. Even though this precluded us from using the same items as in Study 1 , we assessed the same five appraisals and a broad set of eight emotions. English translations of the surveys can be found in the Supplemental Information.

The daily survey consisted of 16 items. First, participants were asked if a specific situation within the past hour had elicited noticeable feelings. If they responded no, they did not see any further questions. Even though this allowed participants to skip the survey by concealing emotional events, the incentive to do so was minimal given that a) the survey was very brief and $b$ ) participants knew that concealing events would bias the personal feedback they were expecting at the end of the study. If they responded yes to the question about an emotional event, they were asked to enter a short title for the event and respond to items assessing emotions, appraisals, and the context of the event.

Emotions. Participants were asked to rate the feelings elicited by the event using 8 items with visual-analog sliders running from "not at all" (invisible value o) to "very strong" (invisible value 100). The items assessed anger, disgust, fear, anxiety, sadness, desire, relaxation, and happiness. Each item was a list of four adjectives (e.g., "irritated, mad, angry, enraged") constructed by translating and concatenating items forming different factors in the Discrete Emotion Questionnaire (Harmon-Jones et al., 2016).

Appraisals. We assessed the same 5 core appraisals as in Study 1. To facilitate faster responding, we reduced the number of items from 10 to 6 by including only one item per appraisal other than congruence where we retained the congruence and incongruence versions. We also altered some items to make them easier to understand and suitable for a broader range of emotional events. See Supplemental Information for explanations and final phrasings. All items were presented with a visual-analog slider running from "do not agree at all" (invisible value o) to "agree completely" (invisible value 100).

Context. We also asked participants to indicate whether the emotional event was related to loved ones; friends or colleagues; strangers; school or work; and leisure or free time.

In the evening survey, participants were shown the short titles they had entered for each emotional event they had reported that day. For each event, they were asked to describe the feelings the event elicited now and the way they appraised it now using the items from the day survey. They were then asked to indicate when the event had started and, if applicable, ended.

Emotion regulation. The remainder of the evening survey assessed different aspects of emotion regulation. Participants were asked whether they had attempted to regulate their emotions in relation to that event. If they had, they were also asked whether they had tried to increase, maintain, or decrease their negative and positive emotions and how successful they had been. Finally, participants identified the emotion regulation strategies they had used from a list of 12 strategies which included two items corresponding to reconstrual reappraisal ("I thought through why this situation was not what it seemed at first") and repurposing reappraisal ("I re-evaluated what this situation has to offer to $\mathrm{me}^{\prime \prime}$ ). 


\section{Results and Discussion}

\section{Preliminary analyses}

Among the final sample of 168 participants, we observed a reasonable response rate across all daily prompts. On average, participants responded to 14.9 out of 21 or $71.2 \%$ (SD = $24.0 \%$ ) of the daily prompts. The response rate declined from $80.1 \%$ on the first day through $74.3 \%, 72.9 \%, 70.5 \% 65.9 \%, 62.9 \%$ to $55.4 \%$ on the last day. An emotional event was reported on average for 7.4 prompts which corresponds to $50.5 \%$ (SD $=23.5 \%)$ of all responded prompts. For $90.6 \%$ of the daily reports (an average of 6.8
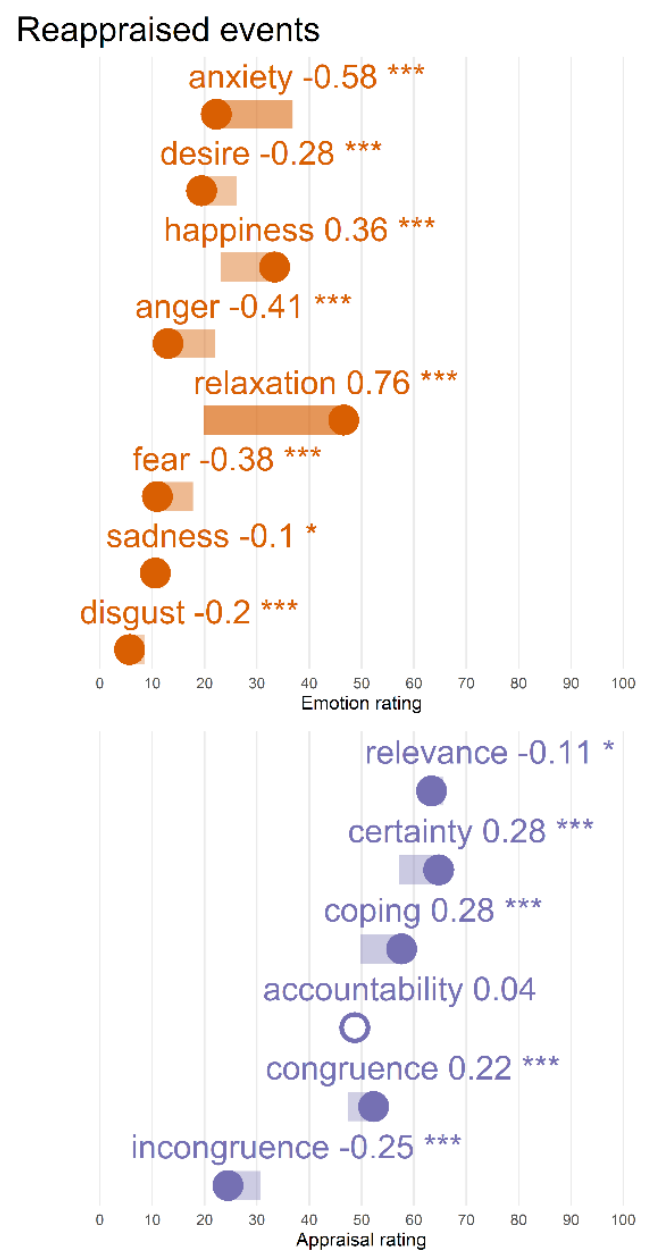

corresponding to $45.6 \%$ of all responded prompts), participants also provided responses in the evening thus yielding 1142 complete responses that form the data analyzed here. On average, participants provided 6.8 full reports $(S D=4.4$, range $2-21)$. Of complete responses, $34.5 \%$ came from the $1^{\text {st }}$ prompt of the day, $33.3 \%$ from the $2^{\text {nd }}$ to $32.2 \%$ from the $3^{\text {rd }}$ prompt of the day, $\chi^{2}(2)=0.89, p=0.64$. The median times at which participants responded to the prompts were at 11:41 for the $1^{\text {st }}$ prompt, at 13:19 for the $2^{\text {nd }}$ prompt, at 15:23 for the $3^{\text {rd }}$ prompt and at 19:30 for the evening prompt.

Unregulated and otherwise regulated events
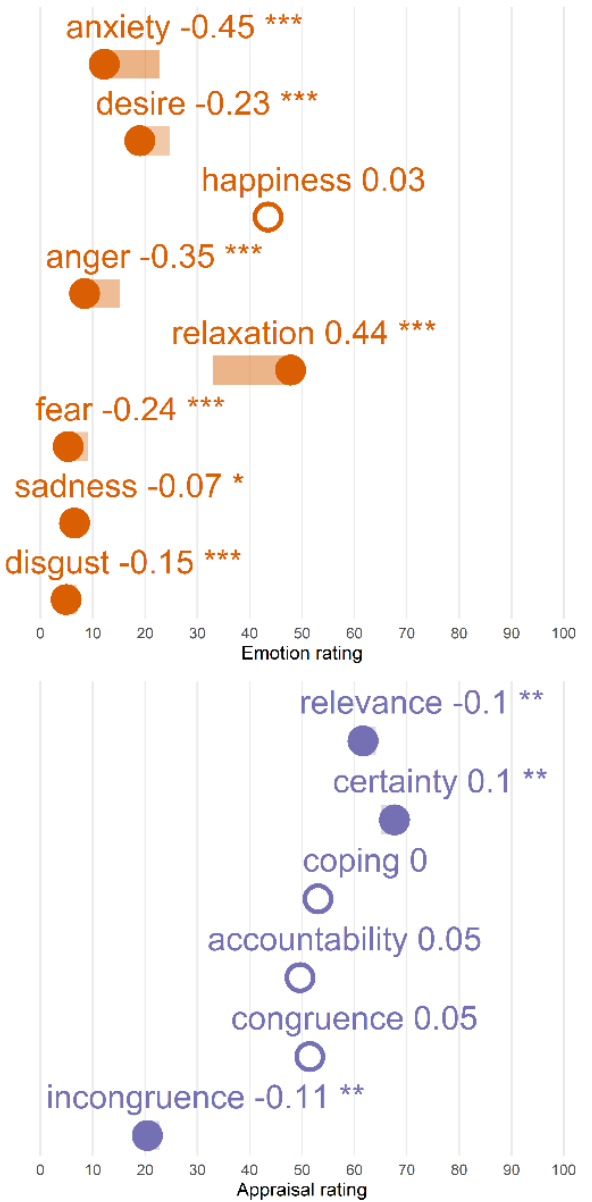

Figure 4. Mean emotion changes (top row) and appraisal shifts (bottom row) from day to evening for events that were reappraised (left column) and not reappraised (right column).

Notes. The beginning of each line depicts day value and the pointed end the evening value on original response scales. Lines are ordered by day values of reappraised events. The numbers depict Cohen's d effect sizes with uncorrected p-values. Filled points depict significant effects and the stars denote significance levels $* * * p<.001, * * p<.01, * p<.05$. 
Next, we analyzed the mean emotion and appraisal ratings from day and evening separately for reappraised and non-reappraised events (see Figure 4 and Supplemental Table 5). For reappraised events $(n=373)$ the reported emotions were regulated using reappraisal either alone $(n=34)$ or in conjunction with other strategies $(n=339)$. For non-reappraised events ( $n=769$ ) the reported emotions were regulated using other strategies $(n=697)$ on not regulated $(n=72)$.

Looking at daily reports we found that mean emotion ratings remained below the scale midpoint and more similar across positive and negative emotions. Thus, on average Study 2 contains less intensely negative and more positive emotions than Study 1 . We also found that participants reported significant changes in these emotions (see Cohen's d effect sizes and ttests in the top row of Figure 4). For reappraised events, all emotions changed significantly from day to evening in the expected direction. For non-reappraised events, emotions changed less albeit still significantly and largely in the expected direction except for happiness which did not change and disgust which increased rather than decreased.

Turning to appraisals, we found that the relevance rating was above the scale midpoint in line with the expectation from appraisal theory that all emotions are characterized by high degree of relevance. Appraisal shifts were more pronounced for reappraised events than for non-reappraised events (see Cohen's $d$ effect sizes and t-tests in the bottom row of Figure 4). For reappraised events, all appraisals other than self-accountability shifted significantly from day to evening. There were small increases in certainty, coping potential, and congruence, a small reduction in incongruence and a very small one in relevance. By contrast, for nonreappraised events, there were only two very small significant shifts, a reduction in relevance and an increase in certainty. Taken together, these results indicate that the data contain significant emotion and appraisal changes that can be related to reappraisal use.

As in Study 1, we used exploratory factor analysis to more parsimoniously represent the inter-correlated variance captured by emotion items. Three factors suggested by parallel analysis were extracted from day data yielding a model with moderate fit $(T L I=0.86$, RMSEA $=$ 0.12). The factors were labelled as satisfied, irritated, and anxious (see Supplemental Table 7 for factor loadings and proportions explained). As in Study 1, factor scores were computed using the regression method for day as well as evening data from the model fit to day data.

Were reappraisal effects on emotion statistically mediated by appraisal shifts?

To test the hypothesis that appraisal shifts statistically mediate reappraisal effects on emotions, we conducted a SEM for each of the three emotion factors (see Figure 5, panel A). In each model, shifts in 5 appraisal dimensions from day to evening were entered as parallel mediators of a change in the emotion factor score controlling for sample-centered mean appraisals. To also learn whether appraisal shifts were more relevant for reappraised compared to non-reappraised events, we estimated the interaction effects between all model paths and the event type (Montoya, 2019). Unlike in Study 1 , the data here are clustered as different observations from the same participant are not independent. Our analyses account for this in two steps. First, the within-subjects mediation model we use removes participant-related variance in raw scores by the use of day to evening difference scores (Hamaker \& Muthén, 2020). Second, to account for the remaining clustering of the difference scores and the sample-centered mean scores by participants, we fit the SEM models with cluster-robust standard errors (Williams, 2000). Detailed results of the mediation analyses can be found in Table 2. Panel B on Figure 5 summarizes the 
key findings by depicting the proportions of change in each emotion that was statistically mediated by appraisal shifts.

Focusing on reappraised events, we found that appraisal shifts statistically mediated $21.8 \%(b=$ $-.11, p<.001)$ of the day-to-evening reduction in feeling anxious, $b=-.52, p<.001$. This emotion change was related to significant reductions in the incongruence $(8.5 \%$ of total emotion change, $p=.042)$ and certainty $(7.8 \%, p=.029)$ appraisals. Moderation analysis suggested that the total indirect effect of appraisal shifts on anxiety was significantly larger for reappraised compared to non-reappraised events (difference of $b=-.09, p=.007$ ).

Appraisal shifts also statistically mediated $46 \%$ $(b=-.11, p=.027)$ of the reduction in feeling irritated, $b=-.23, p<.001$. This emotion change was related to a significant reduction in incongruence appraisal $(26 \%, p=.003)$. The total indirect effect of appraisal shifts on irritation was significantly larger for reappraised compared to non-reappraised events (difference $b=-.10, p=.039$ ).

Finally, appraisal shifts statistically mediated $22.4 \%(b=.11, p<.001)$ of the increase in feeling satisfied, $b=.49, p<.001$. This emotion change was related to a significant increase in congruence appraisal $(6.8 \%, p=.046)$. The total indirect effect of appraisal shifts on satisfaction was significantly larger for reappraised compared to non-reappraised events (difference $b=.10, p=.002$ ).

\section{A}

B
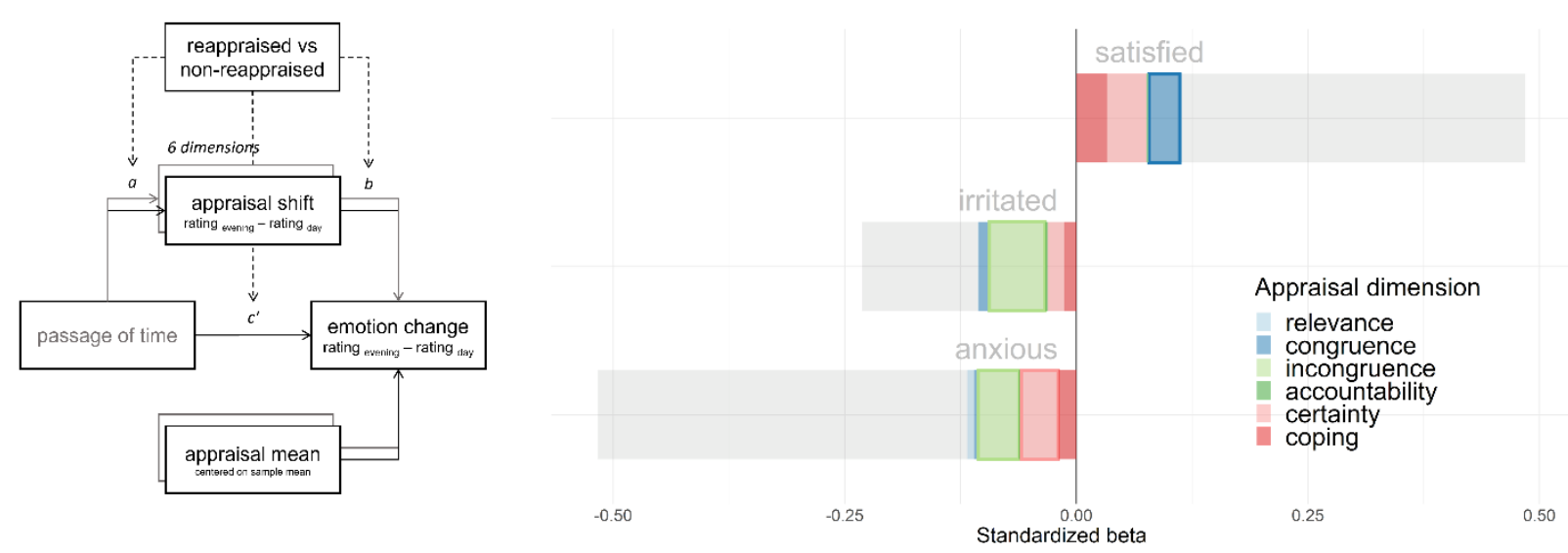

Figure 5. Structure and results of the mediation analyses. Panel A. The two-condition within-subject moderated mediation model. Panel B. Proportions of reappraisal-related emotion changes statistically mediated by appraisal shifts.

Notes. Panel $A$. In the model, emotion change defined as the difference between evening and day emotion factor scores is predicted by 5 appraisal shifts defined as differences between evening and day appraisal ratings, an intercept representing the direct effect passage of time, and 5 appraisal means defined as a sample centered average of evening and day ratings to make the intercept interpretable as a direct effect. The model also included interaction effects between all mediation coefficients and a 2-level factor indicating if the passage of time involved reappraisal or not.

Panel B. Gray bars represent total emotion changes from pre to post regulation. Colored ribbons represent proportions of these changes that were statistically mediated by appraisal shifts. Ribbons with thick outlines denote significant effects at uncorrected $p<.05$. 
Table 2. Standardized path coefficients from 3 within-subjects moderated mediation analyses

\begin{tabular}{|c|c|c|c|c|c|c|}
\hline & \multicolumn{2}{|c|}{ Anxious } & \multicolumn{2}{|c|}{ Irritated } & \multicolumn{2}{|c|}{ Satisfied } \\
\hline & $\beta$ & $p$ & $\beta$ & $\beta$ & $p$ & $p$ \\
\hline \multicolumn{7}{|c|}{ Regulated with reappraisal $(n=373)$} \\
\hline \multicolumn{7}{|l|}{ Appraisal shifts (a) } \\
\hline Relevance & -0.113 & 0.034 & -0.113 & 0.034 & -0.113 & 0.034 \\
\hline Congruence & 0.220 & $<0.001$ & 0.220 & $<0.001$ & 0.220 & $<0.001$ \\
\hline Incongruence & -0.253 & $<0.001$ & -0.253 & $<0.001$ & -0.253 & $<0.001$ \\
\hline Accountability & 0.040 & 0.478 & 0.040 & 0.478 & 0.040 & 0.478 \\
\hline Certainty & 0.284 & $<0.001$ & 0.284 & $<0.001$ & 0.284 & $<0.001$ \\
\hline Coping & 0.285 & $<0.001$ & 0.285 & $<0.001$ & 0.285 & $<0.001$ \\
\hline \multicolumn{7}{|l|}{ Appraisal effects on emotion (b) } \\
\hline Relevance & 0.063 & 0.218 & 0.005 & 0.933 & 0.020 & 0.719 \\
\hline Congruence & -0.023 & 0.707 & -0.054 & 0.441 & 0.152 & 0.008 \\
\hline Incongruence & 0.172 & 0.013 & 0.237 & $<0.001$ & -0.008 & 0.893 \\
\hline Accountability & 0.068 & 0.192 & -0.072 & 0.168 & -0.011 & 0.825 \\
\hline Certainty & -0.140 & 0.013 & -0.062 & 0.293 & 0.151 & 0.050 \\
\hline Coping & -0.069 & 0.213 & -0.046 & 0.388 & 0.116 & 0.038 \\
\hline \multicolumn{7}{|l|}{ Mediated emotion change (a*b) } \\
\hline Relevance & -0.007 & 0.323 & -0.001 & 0.934 & -0.002 & 0.725 \\
\hline Congruence & -0.005 & 0.712 & -0.012 & 0.454 & 0.033 & 0.046 \\
\hline Incongruence & -0.044 & 0.042 & -0.060 & $0.003^{*}$ & 0.002 & 0.893 \\
\hline Accountability & 0.003 & 0.513 & -0.003 & 0.497 & -0.000 & 0.825 \\
\hline Certainty & -0.040 & 0.029 & -0.017 & 0.308 & 0.043 & 0.065 \\
\hline Coping & -0.020 & 0.237 & -0.013 & 0.397 & 0.033 & 0.062 \\
\hline \multicolumn{7}{|l|}{ Total effects } \\
\hline Total mediated emotion change & -0.112 & $<0.001^{*}$ & -0.106 & 0.027 & 0.109 & $<0.001^{*}$ \\
\hline Proportion mediated & 0.218 & & 0.460 & & 0.224 & \\
\hline Unmediated emotion change (c') & -0.404 & $<0.001$ & -0.125 & 0.006 & 0.376 & $<0.001$ \\
\hline Total emotion change & -0.516 & $<0.001$ & -0.231 & $<0.001$ & 0.485 & $<0.001$ \\
\hline \multicolumn{7}{|c|}{ Unregulated and regulated without reappraisal $(n=697)$} \\
\hline \multicolumn{7}{|c|}{ Appraisal shifts (a) } \\
\hline Relevance & -0.103 & 0.005 & -0.103 & 0.005 & -0.103 & 0.005 \\
\hline Congruence & 0.047 & 0.238 & 0.047 & 0.238 & 0.047 & 0.238 \\
\hline Incongruence & -0.113 & 0.003 & -0.113 & 0.003 & -0.113 & 0.003 \\
\hline Accountability & 0.046 & 0.256 & 0.046 & 0.256 & 0.046 & 0.256 \\
\hline Certainty & 0.098 & 0.008 & 0.098 & 0.008 & 0.098 & 0.008 \\
\hline Coping & 0.003 & 0.929 & 0.003 & 0.929 & 0.003 & 0.929 \\
\hline \multicolumn{7}{|l|}{ Appraisal effects on emotion (b) } \\
\hline Relevance & 0.003 & 0.952 & -0.073 & 0.241 & 0.089 & 0.030 \\
\hline Congruence & -0.094 & 0.036 & -0.033 & 0.529 & 0.052 & 0.184 \\
\hline Incongruence & 0.065 & 0.204 & 0.044 & 0.367 & -0.138 & $<0.001$ \\
\hline Accountability & 0.010 & 0.781 & -0.043 & 0.210 & -0.014 & 0.713 \\
\hline Certainty & -0.115 & 0.012 & -0.046 & 0.405 & 0.055 & 0.112 \\
\hline Coping & -0.020 & 0.651 & -0.002 & 0.968 & 0.082 & 0.041 \\
\hline \multicolumn{7}{|l|}{ Mediated emotion change (a*b) } \\
\hline Relevance & -0.000 & 0.952 & 0.008 & 0.221 & -0.009 & 0.078 \\
\hline Congruence & -0.004 & 0.341 & -0.002 & 0.612 & 0.002 & 0.423 \\
\hline Incongruence & -0.007 & 0.210 & -0.005 & 0.396 & 0.016 & 0.013 \\
\hline Accountability & 0.000 & 0.775 & -0.002 & 0.402 & -0.001 & 0.722 \\
\hline Certainty & -0.011 & 0.102 & -0.004 & 0.443 & 0.005 & 0.179 \\
\hline Coping & -0.000 & 0.931 & -0.000 & 0.971 & 0.000 & 0.929 \\
\hline \multicolumn{7}{|l|}{ Total effects } \\
\hline Total mediated emotion change & -0.023 & 0.046 & -0.005 & 0.595 & 0.014 & 0.194 \\
\hline Proportion mediated & 0.058 & & 0.032 & & 0.093 & \\
\hline Unmediated emotion change (c') & -0.376 & $<0.001$ & -0.164 & $<0.001$ & 0.137 & 0.002 \\
\hline Total emotion change & -0.399 & $<0.001$ & -0.169 & $<0.001$ & 0.151 & $<0.001$ \\
\hline \multicolumn{7}{|c|}{ Moderation analysis (reappraised vs non-reappraised) } \\
\hline Relevance mediated & -0.007 & 0.435 & -0.008 & 0.413 & 0.007 & 0.458 \\
\hline Congruence mediated & -0.001 & 0.949 & -0.010 & 0.517 & 0.031 & 0.066 \\
\hline Incongruence mediated & -0.036 & 0.098 & -0.055 & 0.009 & -0.014 & 0.430 \\
\hline
\end{tabular}




\begin{tabular}{rcccccc}
\hline & \multicolumn{2}{c}{ Anxious } & \multicolumn{2}{c}{ Irritated } & \multicolumn{2}{c}{ Satisfied } \\
& $\beta$ & $p$ & $\beta$ & $\beta$ & $p$ & $p$ \\
\hline Accountability mediated & 0.002 & 0.610 & -0.001 & 0.821 & 0.000 & 0.955 \\
Certainty mediated & -0.028 & 0.132 & -0.013 & 0.457 & 0.037 & 0.106 \\
Coping mediated & -0.020 & 0.239 & -0.013 & 0.397 & 0.033 & 0.068 \\
Total mediated & $\mathbf{- 0 . 0 8 9}$ & $\mathbf{0 . 0 0 7 ^ { * }}$ & $\mathbf{- 0 . 1 0 1}$ & $\mathbf{0 . 0 3 9}$ & $\mathbf{0 . 0 9 5}$ & $\mathbf{0 . 0 0 2 ^ { * }}$ \\
Unmediated & -0.028 & 0.507 & 0.039 & 0.662 & $\mathbf{0 . 2 4 0}$ & $\mathbf{0 . 0 0 1}$ \\
\hline
\end{tabular}

Notes. $\beta$ - standardized regression coefficient, $p$ - uncorrected significance value. Bold denotes significant effects at uncorrected $p<0.05$ * denotes $p$-values that remained significant after applying the FDR-correction to the mediation effects on gray background that were relevant for our hypothesis.

The moderated mediation findings support our hypothesis that reappraisal-related emotion changes are statistically mediated by shifts along appraisal dimensions. All appraisal shifts combined had significant small-to-medium effects on all emotion changes associated with reappraisal and explained $22-46 \%$ of their variance. Two out of three of these effects survived the FDR correction. By contrast, appraisal shifts explained only $3.2-9.3 \%$ of the emotion changes associated with nonreappraised events and were less often significant both on the individual dimension and combined levels (see Table 2). These findings indicate that appraisal shifts may indeed be selectively relevant for emotion changes associated with reappraisal.

Can appraisal shift profiles reveal reappraisal tactics?

As in Study 1 , we conducted a latent profile analysis using residualized difference scores reflecting appraisal shifts from day to evening for reappraised events. The class selection criteria suggested a model with 6 classes (see Supplemental Table 7) that are illustrated in Figure 8.

We labelled Class 1 ( $n=15$ reports) "Sour grapes" as it included a large reduction in congruence coupled with a large increase in relaxation. This seemingly paradoxical combination is consistent with a form of reappraisal where the value of a frustrated goal is reduced.

We labelled Class $2(n=16)$ "You never know" as it included a pattern familiar from Study 1 : large reduction in coping and a medium reduction in self-accountability coupled with a very large reduction in incongruence. These shifts were accompanied by a large increase in relaxation, a large reduction in anxiety, and medium reductions in fear and desire.

We labelled the largest Class $3(n=184)$ "Piece of cake". Mastery was implied by a small increase in coping and a very small one in certainty coupled with a small decrease in relevance and a very small one in incongruence. These changes were accompanied by a large increase in relaxation, a large reduction in anxiety, a medium reduction in anger, small reductions in fear and disgust, a small increase in happiness, and a very small reduction in desire.

We labelled Class 4 ( $n=113$ ) "All good" as it included significant shifts across all appraisals: a large increase in congruence, medium increase in certainty, small increases in relevance, coping, and self-accountability, and a small decrease in incongruence. This tactic was accompanied by a very large increase in relaxation and a medium increase in happiness. There were also medium reductions in anxiety, fear, and anger, and a small reduction in desire.

We labelled Class $5(n=28)$ "There will be consequences" as it included very large increases in self-accountability and certainty as well as medium increases in coping and relevance. These shifts were accompanied with small reductions in desire and anger suggesting 
the tactic may involve downregulation of cravings with a focus on personal responsibility.

We labelled Class $6(n=17)$ "Cope to thrive" as it included a familiar pattern from Study 1: a very large increase in coping, a large reduction in self- accountability, a large increase in congruence, and a medium reduction in incongruence. This tactic involved a very large reduction in anxiety, a very large increase in relaxation, and a medium increase in happiness.

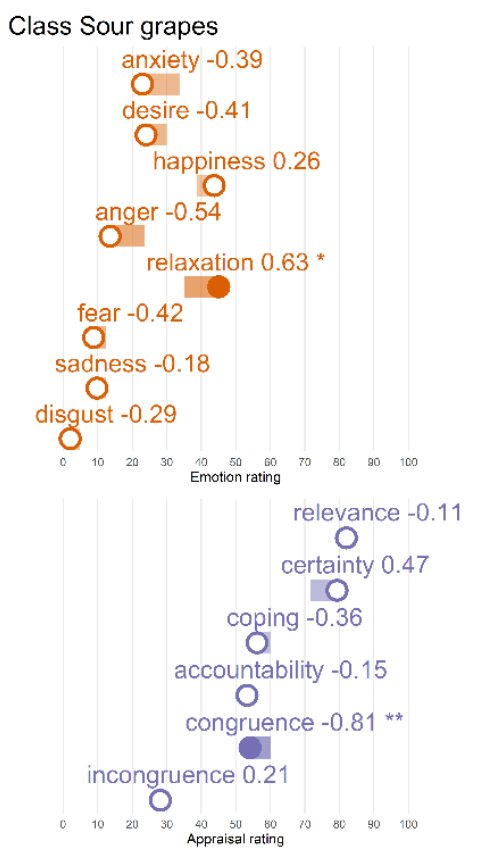

Class All good

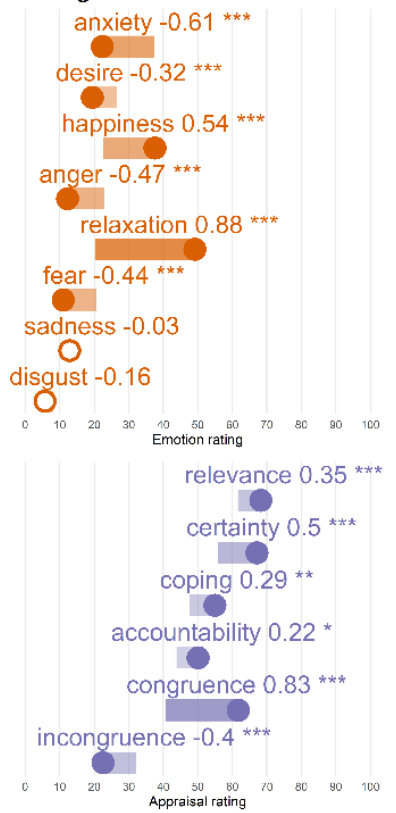

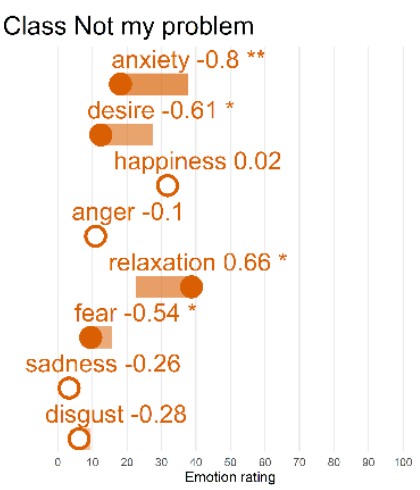

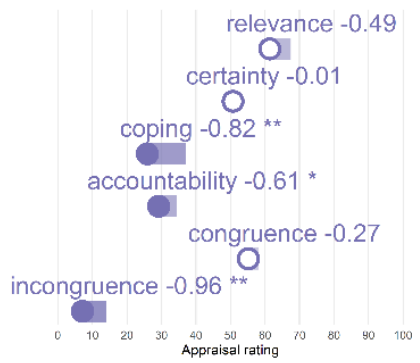

Class There will be consequences
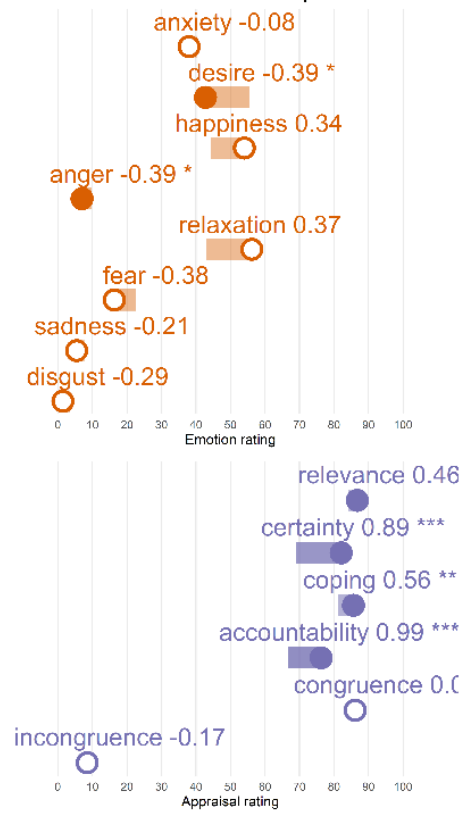
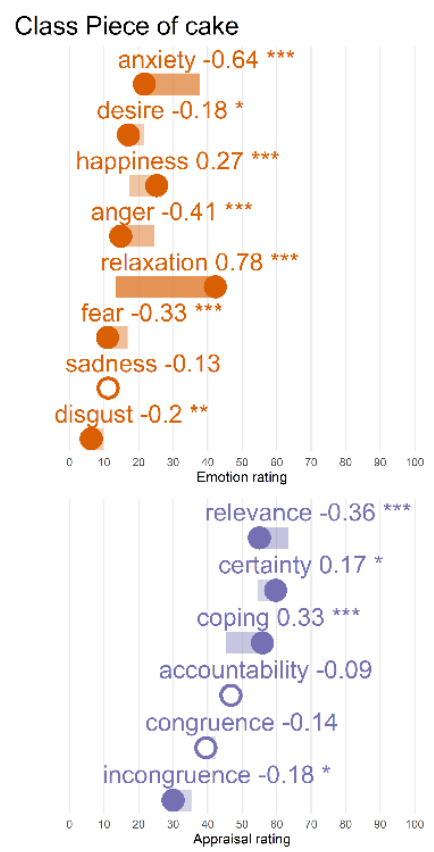

Class Cope to thrive anxiety $-0.89^{* * *}$

desire -0.4

o
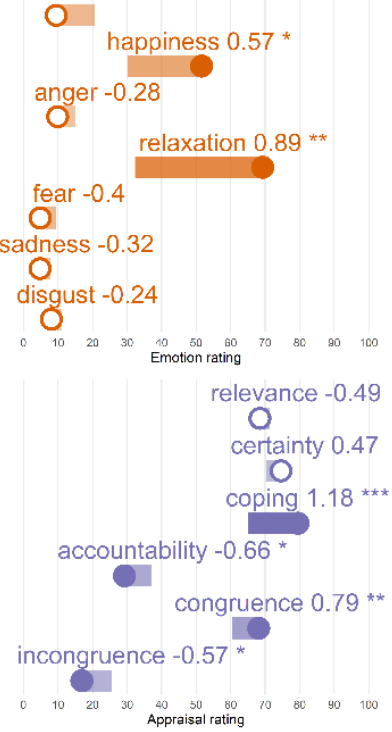

Figure 8. Mean appraisal shifts and emotion changes across reappraisal classes.

Notes. The beginning of each line depicts pre-regulation value and the pointed end the post-regulation value on original response scales. Lines are ordered by pre-regulation values. The numbers depict Cohen's D effect sizes with uncorrected $p$ values. Filled points depict significant effects and the stars denote significance levels $* * * p<.001, * * p<.01, * p<.05$. 
Taken together, the latent class analyses suggest that as in Study 1, appraisal shift profiles can reveal distinct and conceptually coherent clusters that can be seen as reappraisal tactics. Unlike in Study 1, no appraisal dimension was significantly changed in the same direction in all classes. This can be explained by the higher variance in the valence and arousal of events reappraised in Study 2 . This finding further reiterates the value of using a multivariate profile to characterize reappraisal.

Using a variance components analysis explained in Supplemental Information, we also found that the appraisal shift profiles were not primarily reflections of stable individual differences nor very broad features of situations. Instead, they appeared to capture the interplay between an individual and a situation. This is consistent with the idea that appraisal dimensions reflect the motivational meaning of a situation which is a function of the situation as well as the motives an individual brings to the situation.

\section{Interim summary}

As in Study 1, we found support for the hypothesis that appraisal shifts statistically mediate reappraisal-related emotion change. Extending Study 1 , here we also found that the mediating role of appraisal shifts may be relatively specific to reappraisal. Even though emotions changed from day to evening also without reappraisal and even though a few appraisal shifts also mediated such changes, these changes and in particular these mediation effects were clearly smaller for non-reappraised compared to reappraised events. Given that for most reappraised events participants used reappraisal together with other strategies, the reappraised-related appraisal shift effects may in principle include additional benefits from other regulation strategies. However, these benefits were presumably also present in the otherwise regulated events that formed the bulk of non-reappraised events. The clear contrast between the statistical mediation of appraisal shifts between reappraised and nonreappraised events thus continues to support their hypothesized mechanistic role.

Study 2 also further demonstrated the potential of appraisal shift profiles to reveal different reappraisal tactics. With two exceptions, the reappraisal classes found in this study were different from the ones in Study 1 . This is not surprising given that in Study 1 participants selected one of the worst events from the past 2 months whereas in Study 2 they selected the most intense event from the past hour which did not have to be negative. The discovery of six distinct classes, four of which were unique to Study 2, therefore indicates the flexibility of using appraisal shift profiles to capture reappraisal variance.

Overall, Study 2 suggests that the mediating role of appraisals and the flexibility of appraisal shift profiles in capturing distinct forms of reappraisal were not dependent on methodological limitations of Study 1 . The current design was less sensitive to memory biases as the day reports were given within an hour of the event. It also mitigated demand characteristics by asking for evening reports several hours later even though participants' awareness of the aims of the study may still have somewhat biased their responses. Also, given that participants were free to choose whether and how to regulate emotion, the current findings confound the effects of using reappraisal with the effects of deciding to do so. For instance, people are known to prefer distraction to reappraisal when viewing affective images eliciting high compared to low intensity negative emotions (Sheppes, 2014). Regulatory choice processes may thus explain at least some of the differences between reappraised and non-reappraised experiences. 


\section{GENERAL DISCUSSION}

Reappraisal occupies a central position among the strategies people use to regulate emotion (Gross, 1998). It is known to be effective in altering the subjective, physiological, and neural components of emotion (Webb et al., 2012) and, over the long run, facilitating mental health (Aldao et al., 2010; Lincoln, 2022). However, less is known about the cognitive mechanisms underlying reappraisal, perhaps because analysis of thought content is challenging. The reAppraisal framework suggests that operationalizing reappraisals as profiles of appraisal shifts can be an important step towards learning more about reappraisal mechanisms (Uusberg et al., 2019). The present research was designed to test the validity as well as feasibility of this approach. In two studies, participants used reappraisal to successfully reduce negative as well as increase positive emotions. They also rated their emotions and appraisals both before and after engaging in reappraisal allowing us to ask two questions: Did appraisal shifts statistically mediate reappraisalrelated emotion changes? Can appraisal shifts function as a coordinate space for instances of reappraisal?

\section{Did Appraisal Shifts Statistically Mediate Reappraisal-related Emotion Changes?}

We found strong support for the hypothesis that appraisal shifts statistically mediate reappraisalrelated emotion changes. In Study 1, the ten appraisal shifts modelled as parallel mediators collectively explained between $19 \%$ and $49 \%$ of changes in different emotions from before to after using reappraisal. In Study 2, the six appraisal shifts modelled as parallel mediators collectively explained a similar proportion of $22 \%$ to $46 \%$ of variance in emotion changes from day to evening in reports where reappraisal was used. By contrast, appraisal shifts were significantly less relevant for reports with no reappraisal.
These findings are consistent with viewing appraisal shifts as a mechanism through which emotions change during reappraisal. In isolation, statistical mediation does not prove mechanistical mediation unless the data meet requirements for causal evidence such as assessment of the mediator (appraisal shift) and outcome (feeling change) at different time points (Fiedler et al., 2011). As these conditions were not met in the present data, the significant statistical mediation effect may reflect the hypothesized causal path (appraisal shifts cause feeling changes), but also a reverse path (feeling changes cause appraisal shifts), or a third variable causing both. Although we cannot rule out the latter two interpretations, there are conceptual and empirical reasons to doubt they could explain all of the present findings. Conceptually, even as most theories acknowledge bidirectional influences between different components of emotion, few theories would deny that appraisals can be a source of such influences (Moors, 2022). To the contrary, most authors consider some form of appraisal to be a key driver of variance in other emotion components (Moors, 2013). Thus, even if some portion of the mediation effect observed in this study should be attributed to other causal pathways, at least some of it probably reflects appraisal shifts causing emotion changes.

A further empirical reason to consider the statistical mediation observed here as a probable indication of underlying mechanistic mediation is provided by Study 2. Presumably, if appraisal shifts were caused by changes in other emotion components and not the other way around, then appraisal shifts should be quite similar across emotion changes that accompany reappraisal and emotion changes that accompany other forms of regulation or spontaneous habituation. However, this is not what we found in Study 2. While some appraisal shifts correlated with emotion changes observed at the absence of reappraisal, these explained less emotion change variance than 
appraisal shifts observed at the presence of reappraisal. For these reasons, even though the statistical mediation effects of this study cannot prove the role of appraisal shifts in reappraisal, we believe it is unlikely that the bulk of these effects could be explained by other mechanisms.

One may also wonder why appraisal shifts did not explain more of the emotion change variance. While it is possible that reappraisal also works through pathways other than appraisal shifts, it is also possible the proportions we observed underestimate the true proportions of emotion changes mediated by appraisal shifts. First, some of the emotion changes might have been mediated by shifts in appraisals that we did not assess. The five most common appraisals assessed here are not an exhaustive list of all appraisal dimensions that can matter for emotions (Moors et al., 2013). Second, to keep the mediation models reasonably simple, we included appraisal main effects but not any of their interactions. It is likely that some of the emotion variance was mediated by combinations of two or more appraisal dimensions. This was in fact suggested by the LPA results demonstrating that significant emotion changes were associated with different appraisal shift profiles. Finally, observed correlations are inevitably capped by measurement reliability which was only moderate for appraisals. Therefore, it is quite likely that the present findings provide a conservative estimate of the true proportion of reappraisal-related emotion change that is statistically mediated by appraisal shifts.

\section{Can Appraisal Shifts Function as a Coordinate Space for Instances of Reappraisal?}

The second proposition tested in the present research is that appraisal shift profiles can be used to represent distinct forms of reappraisal such as different reappraisal tactics. A multidimensional space offers a flexible way to systematically characterize highly variable phenomena. For example, millions of colors can be represented as a profile of three values on the red, green, and blue dimensions. Can appraisal shift profiles do to reappraisal what systems like RGB have done to color representation?

Our findings from the latent profile analyses support a tentative "yes". The variance in appraisal shift profiles contained four and six distinct clusters in Studies 1 and 2, respectively. Finding more than one cluster suggests that expressing reappraisals as appraisal shift profiles can unearth similarities between otherwise idiosyncratic thought processes. We also found that the appraisal shifts and emotion changes were coherent within a class and distinct between classes. Given that each class was associated with significant improvements in emotion, these findings suggest that successful reappraisal was achieved through different appraisal shift combinations.

Furthermore, we found considerable variance in the nature of helpful appraisal shifts. In Study $\mathbf{1}_{\text {, }}$ perhaps owing to the more homogenous set of emotions being reappraised, we found that reducing relevance and incongruence and increasing outlook valence and emotional coping potential were part of each helpful tactic. However, the remaining dimensions in Study 1 and all dimensions measured in Study 2 could shift as well as not shift or shift in one direction as well as in another within different tactics. On a substantive level, this pattern is an important observation about how people use reappraisal suggesting that the world of potentially helpful cognitive changes is quite diverse. On a methodological level, it underscores the value of taking a multivariate rather than univariate approach to assessing instances of reappraisal.

In Study 2, we also found that the probability of belonging to a given appraisal shift class was not determined by individual differences or situational features in isolation, but instead by their interaction. This suggests that appraisal shift profiles can capture variance at the 
intersection between individual and situation, where the potential for reappraisal indeed should lie. Taken together, these findings make us hopeful that appraisal shift profiles may provide a promising approach to capturing reappraisal tactics as well as single instances of reappraisal.

\section{Implications for Emotion Research and Interventions}

The present findings begin to fill an important gap in the field of emotion research. Demonstrating how intentional cognitive change leads to emotion change through appraisal shifts is a missing link between two sets of existing findings. The first set demonstrates how manipulated and observed reappraisal use leads to changes in components of emotion (Webb et al., 2012). The second set demonstrates how manipulated and observed appraisal changes can impact the same components (Scherer \& Moors, 2019). The present research links these two lines of inquiry by showing how reappraisal can shift appraisals in a way that can change the subjective feeling component of emotion.

The usefulness of appraisal shifts as a model of reappraisal need not end with research. We can imagine psychoeducational interventions encouraging individuals to cycle through appraisal dimensions when they engage in emotion regulation. For instance, people could be trained to ask a series of questions as a way to catalyze appraisal shifts along specific dimensions. In the context of negative emotions, relevance shifts could be catalyzed by asking "Is the situation really as important as it seems?"; congruence shifts by asking "Could this situation have a silver lining?"; accountability shifts by asking "Maybe I'm blaming myself or others too much?"; certainty shifts by asking "How sure am I that I know what is really happening?"; and coping potential shifts by asking "Might I be underestimating the ways in which I can deal with this situation?".

\section{Limitations and Future Directions}

The limitations of these studies begin with the reliance on self-reports which introduced biases, demand characteristics, and prevented us from assessing appraisal shifts before they lead to emotion changes and emotion changes only after that. Future research could address this limitation by adopting psychophysiological correlates of appraisals such as neural activation or gaze patterns in relation to carefully designed stimuli. Another approach would be directly manipulate appraisal shifts in laboratory settings. Somewhat further off, computational linguistic models could identify appraisals from natural language (Troiano et al., 2022). Another important limitation is the reliance of reactivated emotions in Study 1 which should not be equated with concurrent emotions. Even if Study 2 suffered less from this limitation, future research is needed to establish the generalizability of the current findings, including to larger and more diverse samples. Future work could also extend the current approach to a broader set of appraisal dimensions.

\section{Conclusions}

In a pair of complementary studies, we found that appraisal shifts statistically mediate the effects that manipulated and observed use of reappraisal has on emotion. Taken together with emotion theories, these findings suggest, although they do not prove, that appraisal shifts are a key mechanism through which changes to thinking about an emotion situation can lead to changes in emotions. We also illustrated how appraisal shift profiles can provide a succinct and flexible coordinate space for representing instances of reappraisal. We hope these findings encourage other researchers to use appraisal shift profiles to model the cognitive processes underlying reappraisal. 


\section{REFERENCES}

Aldao, A., Nolen-Hoeksema, S., \& Schweizer, S. (2010). Emotion-regulation strategies across psychopathology: A meta-analytic review. Clinical Psychology Review, 30(2), 217-237. https://doi.org/10.1016/j.cpr.2009.11.004

Buhle, J. T., Silvers, J. A., Wager, T. D., Lopez, R., Onyemekwu, C., Kober, H., Weber, J., \& Ochsner, K. N. (2013). Cognitive reappraisal of emotion: A meta-analysis of human neuroimaging studies. Cerebral Cortex, bht154. https://doi.org/10.1093/cercor/bht154

Carver, C. S., Scheier, M. F., \& Weintraub, J. K. (1989). Assessing coping strategies: A theoretically based approach. Journal of Personality and Social Psychology, 56(2), 267.

Castro-Schilo, L., \& Grimm, K. J. (2018). Using residualized change versus difference scores for longitudinal research. Journal of Social and Personal Relationships, 35(1), 32-58. https://doi.org/10.1177/0265407517718387

Fiedler, K., Schott, M., \& Meiser, T. (2011). What mediation analysis can (not) do. Journal of Experimental Social Psychology, 47(6), 1231-1236. https://doi.org/10.1016/j.jesp.2011.05.007

Funder, D. C., \& Ozer, D. J. (2019). Evaluating Effect Size in Psychological Research: Sense and Nonsense. Advances in Methods and Practices in Psychological Science, 2515245919847202. https://doi.org/10.1177/2515245919847202

Garnefski, N., \& Kraaij, V. (2007). The Cognitive Emotion Regulation Questionnaire. European Journal of Psychological Assessment, 23(3), 141-149. https://doi.org/10.1027/1015-5759.23·3.141

Gross, J. J. (1998). The emerging field of emotion regulation: An integrative review. Review of General Psychology, 2(3), 271-299.

Gross, J. J. (2015). Emotion regulation: Current status and future prospects. Psychological Inquiry, 26(1), 126.

https://doi.org/10.1080/1047840X.2014.940781

Gross, J. J., \& John, O. P. (2003). Individual differences in two emotion regulation processes: Implications for affect, relationships, and well-being. Journal of Personality and Social Psychology, 85(2), 348362.

Hamaker, E. L., \& Muthén, B. (2020). The fixed versus random effects debate and how it relates to centering in multilevel modeling. Psychological Methods, 25(3), 365-379. https://doi.org/10.1037/metooo0239

Harmon-Jones, C., Bastian, B., \& Harmon-Jones, E. (2016). The Discrete Emotions Questionnaire: A New Tool for Measuring State Self-Reported
Emotions. PLOS ONE, 11(8), e0159915. https://doi.org/10.1371/journal.pone.0159915

Haynes, T. L., Heckhausen, J., Chipperfield, J. G., Perry, R. P., \& Newall, N. E. (2009). Primary and Secondary Control Strategies: Implications for Health and Well-Being Among Older Adults. Journal of Social and Clinical Psychology, 28(2), 165-197. https://doi.org/10.1521/jscp.2009.28.2.165

Koo, T. K., \& Li, M. Y. (2016). A Guideline of Selecting and Reporting Intraclass Correlation Coefficients for Reliability Research. Journal of Chiropractic Medicine, 15(2), 155-163. https://doi.org/10.1016/j.jcm.2016.02.012

Lazarus, R. S. (1968). Emotions and adaptation: Conceptual and empirical relations. Nebraska Symposium on Motivation, 16, 175-266.

Lincoln, T. M. (2022). The role of emotion regulation in the characterization, development and treatment of psychopathology. 15.

McRae, K. (2016). Cognitive emotion regulation: A review of theory and scientific findings. Current Opinion in Behavioral Sciences, 10, 119-124. https://doi.org/10.1016/j.cobeha.2016.06.004

McRae, K., Ciesielski, B., \& Gross, J. J. (2012). Unpacking cognitive reappraisal: Goals, tactics, and outcomes. Emotion, 12(2), 250-255. https://doi.org/10.1037/a0026351

Montoya, A. K. (2019). Moderation analysis in two-instance repeated measures designs: Probing methods and multiple moderator models. Behavior Research Methods, 51(1), 61-82. https://doi.org/10.3758/s13428-018-1088-6

Montoya, A. K., \& Hayes, A. F. (2017). Two-condition within-participant statistical mediation analysis: A path-analytic framework. Psychological Methods, 22(1), 6-27. https://doi.org/10.1037/metooo0086

Moors, A. (2013). On the causal role of appraisal in emotion. Emotion Review, 5(2), 132-140. https://doi.org/10.1177/1754073912463601

Moors, A. (2022). Demystifying Emotions: A Typology of Theories in Psychology and Philosophy (1st ed.). Cambridge University Press. https://doi.org/10.1017/9781107588882

Moors, A., Ellsworth, P. C., Scherer, K. R., \& Frijda, N. H. (2013). Appraisal theories of emotion: State of the art and future development. Emotion Review, 5(2), https://doi.org/10.1177/1754073912468165

Nylund, K. L., Asparouhov, T., \& Muthén, B. O. (2007). Deciding on the Number of Classes in Latent Class Analysis and Growth Mixture Modeling: A Monte Carlo Simulation Study. Structural 
Equation Modeling: A Multidisciplinary Journal, 14(4), 535-569. https://doi.org/10.1080/10705510701575396

R Core Team. (2014). R: A language and environment for statistical computing. R Foundation for Statistical Computing. http://www.R-project.org/

Revelle, W. (2014). Package 'psych.' R Package Version, 1(2.3).

ftp://193.206.139.34/pub/1/cran/web/packages/p sych/psych.pdf

Rosenberg, J., Beymer, P., Anderson, D., van Lissa, C. j., \& Schmidt, J. (2018). tidyLPA: An R Package to Easily Carry Out Latent Profile Analysis (LPA) Using Open-Source or Commercial Software. Journal of Open Source Software, 3(30), 978. https://doi.org/10.21105/joss.00978

Rosseel, Y. (2012). lavaan: An R package for structural equation modeling. Journal of Statistical Software, 48(2), 1-36.

RStudio Team. (2015). RStudio: Integrated development for R. RStudio, Inc., Boston, MA. URL Http://WwW. RStudio. Com/lde.

Scherer, K. R., \& Moors, A. (2019). The Emotion Process: Event Appraisal and Component Differentiation. Annual Review of Psychology, 70(1). https://doi.org/10.1146/annurev-psych-122216011854

Scherer, K. R., Schorr, A., \& Johnstone, T. (Eds.). (2001). Appraisal processes in emotion: Theory, methods, research (Vol. xiv). Oxford University Press.

Sheppes, G. (2014). Emotion regulation choice: Theory and findings. In J. J. Gross (Ed.), Handbook of emotion regulation (Second Edition, pp. 126-139). The Guilford Press.

Smith, C. A., \& Lazarus, R. S. (1993). Appraisal components, core relational themes, and the emotions. Cognition \& Emotion, 7(3-4), 233-269.

Suri, G., Sheppes, G., Young, G., Abraham, D., McRae, K., \& Gross, J. J. (2018). Emotion regulation choice: The role of environmental affordances. Cognition and Emotion, 32(5), 963-971. https://doi.org/10.1080/02699931.2017.1371003

Suri, G., Whittaker, K., \& Gross, J. J. (2014). Launching Reappraisal: It's Less Common Than You Might Think. Emotion, No Pagination Specified. https://doi.org/10.1037/emooo00011

Troiano, E., Oberländer, L., \& Klinger, R. (2022). Dimensional Modeling of Emotions in Text with
Appraisal Theories: Corpus Creation, Annotation Reliability, and Prediction (arXiv:2206.05238). arXiv. http://arxiv.org/abs/2206.05238

Uusberg, A., Taxer, J. L., Yih, J., Uusberg, H., \& Gross, J. J. (2019). Reappraising reappraisal. Emotion Review, 11(4), 267-282. https://doi.org/10.1177/1754073919862617

Vishkin, A., Hasson, Y., Millgram, Y., \& Tamir, M. (2020). One Size Does Not Fit All: Tailoring Cognitive Reappraisal to Different Emotions. Personality and Social Psychology Bulletin, 46(3), 469-484. https://doi.org/10.1177/0146167219861432

Wang, K., Goldenberg, A., Dorison, C. A., Miller, J. K., Uusberg, A., Lerner, J. S., Gross, J. J., Agesin, B. B., Bernardo, M., Campos, O., Eudave, L., Grzech, K., Ozery, D. H., Jackson, E. A., Garcia, E. O. L., Drexler, S. M., Jurković, A. P., Rana, K., Wilson, J. P., ... Moshontz, H. (2021). A multicountry test of brief reappraisal interventions on emotions during the COVID-19 pandemic. Nature Human Behaviour, 1-22. https://doi.org/10.1038/s41562-021-01173-X

Webb, T. L., Miles, E., \& Sheeran, P. (2012). Dealing with feeling: A meta-analysis of the effectiveness of strategies derived from the process model of emotion regulation. Psychological Bulletin, 138(4), 775-808. https://doi.org/10.1037/a0027600

Wickham, H., Averick, M., Bryan, J., Chang, W., McGowan, L. D., François, R., Grolemund, G., Hayes, A., Henry, L., Hester, J., Kuhn, M., Pedersen, T. L., Miller, E., Bache, S. M., Müller, K., Ooms, J., Robinson, D., Seidel, D. P., Spinu, V., ... Yutani, $\mathrm{H}$. (2019). Welcome to the Tidyverse. Journal of Open Source Software, 4(43), 1686. https://doi.org/10.21105/joss.01686

Williams, R. L. (2000). A Note on Robust Variance Estimation for Cluster-Correlated Data. Biometrics, 56(2), 645-646. https://doi.org/10.1111/j.0006$341 X .2000 .00645 \cdot X$

Yih, J., Kirby, L. D., Spitzer, E. G., \& Smith, C. A. (2019). Emotion as a process: Appraisal, emotion, and coping patterns across time. Motivation Science. https://doi.org/10.1037/motooo0144

Yih, J., Uusberg, A., Taxer, J. L., \& Gross, J. J. (2019). Better together: A unified perspective on appraisal and emotion regulation. Cognition and Emotion, 33(1), 41-47. https://doi.org/10.1080/02699931.2018.1504749 